ESAIM: M2AN 46 (2012) 1201-1224

DOI: $10.1051 / \mathrm{m} 2 \mathrm{an} / 2012001$
ESAIM: Mathematical Modelling and Numerical Analysis

www.esaim-m2an.org

\title{
COMPUTATION OF THE DRAG FORCE ON A SPHERE CLOSE TO A WALL
}

\author{
THE ROUGHNESS ISSUE
}

\author{
DAVID GÉRARD-VARET ${ }^{1}$ AND Matthieu Hillairet ${ }^{2}$
}

\begin{abstract}
We consider the effect of surface roughness on solid-solid contact in a Stokes flow. Various models for the roughness are considered, and a unified methodology is given to derive the corresponding asymptotics of the drag force in the close-contact limit. In this way, we recover and clarify the various expressions that can be found in previous studies.
\end{abstract}

Mathematics Subject Classification. 35Q35, 35Q30, 74F10.

Received March 13, 2011. Revised October 14, 2011.

Published online March 15, 2012.

\section{INTRODUCTION}

The dynamics of solid particles in a viscous fluid is crucial to many phenomena, such as blood flow, sedimentation or filtration. The drag force exerted by the fluid on the solids plays of course a central role in this dynamics. It has been the matter of many theoretical studies, and has motivated recently several numerical works (see [19] for a review). The first studies focused on the dynamics of a rigid sphere near a plane wall, that moves in a Stokes flow under no-slip conditions: we refer to the pioneering works $[5,7,21,22]$. The main conclusion of these works is that the drag force is inversely proportional to the distance $h=h(t)$ between the sphere and the plane at time $t$. The reduced ordinary differential equation that governs the movement of the sphere is then of the type: $\ddot{h}+\dot{h} / h=f$, which prevents collision between the sphere and the wall in finite time. We quote that this striking conclusion holds for any value of the fluid viscosity and of the sphere density. Moreover, it is still valid for arbitrary solids with smooth surfaces, and it is still valid within an unsteady Navier-Stokes flow (see [12]).

This theoretical no-collision result, which goes against Archimedes' principle, is clearly unrealistic at the scale of macroscopic solids. Even at microscopic scales, "dry collisions" have been clearly recognized. Therefore, many articles have tried to identify the flaw of the previous modelling, in order to circumvent the paradox. Among possible flaws that have been suggested one can mention:

- the rigidity assumption. Elasticity, even weak could allow for solid contact: see [9];

- the no-slip condition, that is no longer valid when the distance between the solids is of the order of the mean free path of the fluid particles: see [13];

- the incompressibility assumption: see [2].

\footnotetext{
Keywords and phrases. Fluid mechanics, Stokes equations, drag, roughness, homogenization, Navier boundary condition.

1 Institut de Mathématiques de Jussieu, 175 rue du Chevaleret, 75013 Paris, France. gerard-varet@math.jussieu.fr

2 Ceremade, Place du Maréchal de Lattre de Tassigny, 75775 Paris Cedex 16, France. hillairet@ceremade.dauphine.fr
} 


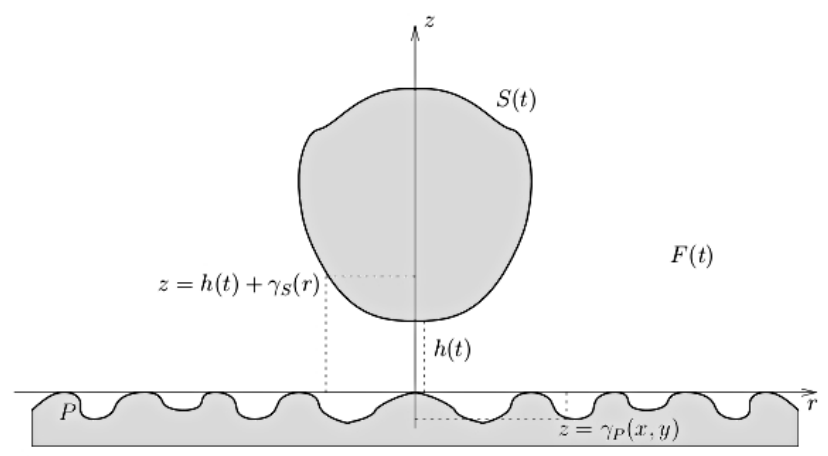

Figure 1. General notations.

We shall focus here on another very popular explanation for the no-collision paradox: roughness. The basic idea is that nothing is as smooth as a plane or a sphere: irregularity of the surface can thus affect the fluid-solid interaction. This credo has led to many experimental and theoretical studies, focusing on roughness-induced effects on drag forces $([8,18,23])$. Such studies will be discussed in the core of the paper.

We quote that the interest in roughness issues has been renewed these last years, notably in connection to microfluidics. Indeed, it has been recognized that the classical no-slip boundary condition, which is relevant at the macroscopic scale, may fail at the micro- or nanoscale. This happens for instance for some corrugated hydrophobic surfaces, which trap gas bubbles in their humps and generate in this way substantial slip. More generally, to determine the appropriate boundary condition at a rough surface is a matter of current debate. In this context, if one has theoretical formulas that express how the drag force depends on the "rough" boundary conditions, one may check experimentally through the force measurement what the right boundary condition is. This interesting point of view is for instance developed in [16,24].

The aim of this paper is to investigate mathematically and in a unified way the relation between the roughness and the drag force. Namely, we study the evolution with time $t$ of a rough solid $S(t)$, falling towards a rough wall $P$ in a Stokes flow. We assume for simplicity that the solid moves by translating along the vertical axis $r=0$, where $(r, \theta, z)$ are cylindrical coordinates. We shall comment on this simplification later on. Various models for the roughness are to be considered. In all models, the moving solid is described at time $t$ by $S(t)=h(t)+S$ for a fixed $S$. We assume that $S$ has its lower tip at $r=0$, and that in the vicinity of its lower tip, its surface is described by:

$$
z=\gamma_{S}(r), \quad r \leq r_{0}, \quad \theta \in(0,2 \pi)
$$

for some $r_{0} \leq 1$ and some Lipschitz function $\gamma_{S}$ with $\gamma_{S}(0)=0, \gamma_{S} \geq 0$. Notice that the solid velocity is given by $\dot{h}(t) e_{z}$. Similarly, the wall $P$ is described in cartesian coordinates $(x, y, z)$, by

$$
z=\gamma_{P}(x, y), \quad(x, y) \in \mathbb{R}^{2},
$$

for some Lipschitz function $\gamma_{P}$, with $\gamma_{P}(0,0)=0, \gamma_{P} \leq 0$. Accordingly, we denote the fluid domain

$$
F(t):=\left\{\mathrm{x}=(x, y, z), \quad \mathrm{x} \notin \overline{S(t)}, \quad z>\gamma_{P}(x, y)\right\}
$$

All these notations are summarized in Figure 1.

If $u=u(t, \mathrm{x})=\left(u_{x}(t, \mathrm{x}), u_{y}(t, \mathrm{x}), u_{z}(t, \mathrm{x})\right)$ and $p=p(t, \mathrm{x})$ stand for the fluid velocity and pressure, the steady Stokes equations read

$$
-\Delta u+\nabla p=0, \quad \operatorname{div} u=0, \quad t>0, \mathrm{x} \in F(t) .
$$


We neglect gravity, as it plays no role in the discussion. Our goal is to study the force on the sphere, that is

$$
\mathcal{F}_{d}(t):=\int_{\partial S(t)}(2 D(u) n-p n) \mathrm{d} \sigma \cdot e_{z} .
$$

The notations $n$ and $D(u)$ refer to the normal vector pointing outside the fluid domain and the symmetric part of the gradient respectively.

In order to determine $\mathcal{F}_{d}(t)$, one needs to specify the boundary conditions at the solid surface and at the plane. In all our models of roughness, such conditions have the following general form:

$$
\left.\left(u-\dot{h}(t) e_{z}\right) \cdot n\right|_{\partial S(t)}=0, \quad\left(u-\dot{h}(t) e_{z}\right) \times\left. n\right|_{\partial S(t)}=-2 \beta_{S}[D(u) n] \times\left. n\right|_{\partial S(t)}
$$

and

$$
\left.u \cdot n\right|_{P}=0, \quad u \times\left. n\right|_{P}=-2 \beta_{P}[D(u) n] \times\left. n\right|_{P}
$$

where $\beta_{S}, \beta_{P} \in[0,+\infty)$. These are boundary conditions of Navier type, the constants $\beta_{P}$ and $\beta_{S}$ being the slip lengths. Of special importance is the case $\beta_{S}=\beta_{P}=0$, which corresponds to the no-slip condition.

We model the roughness in three different ways (pictured in Fig. 2):

1. through a lack of differentiability. Namely, we consider a solid $S$ which is axisymmetric around $\{r=0\}$, and satisfies

$$
\gamma_{S}(r):=1-\sqrt{1-r^{2}}+\varepsilon r^{1+\alpha}, \quad \alpha \in[0,1), \quad r \leq r_{0} .
$$

This means that the solid surface is locally a smooth sphere $z=1-\sqrt{1-r^{2}}$, perturbed by a less regular "rough profile" of amplitude $\varepsilon$. For $\alpha=0$, this profile is a spike, which has Lipschitz regularity. For $\alpha>0$, the profile is differentiable, with a Hölder derivative. For simplicity, we do not consider any roughness on the wall, and take the classical no-slip boundary conditions: $\gamma_{P}=0, \beta_{P}=\beta_{S}=0$;

2. through a slip condition. We consider the case of a ball $S$, of radius 1 , falling vertically above a plane wall, with positive slip coefficients:

$$
\gamma_{S}(r)=1-\sqrt{1-r^{2}}, \quad \gamma_{P}=0, \quad \beta_{S}, \beta_{P}>0 .
$$

Let us stress that such modelling of the roughness by the addition of (small) slip is commonly used. It is well-accepted in the context of rough hydrophobic surfaces [4], and a topic of debate in the context of hydrophilic ones $c f .[16,24]$;

3. through a small parameter. Namely, the roughness is modelled through a small amplitude, high frequency perturbation of a plane wall. That means $P$ is described by the equation

$$
z=\gamma_{P}(x, y):=\varepsilon \gamma(x / \varepsilon, y / \varepsilon), \quad \varepsilon \ll 1
$$

for some periodic and smooth non-positive function $\gamma(X, Y)$, with $\gamma(0,0)=0$. In parallel, we assume that there is no roughness on the solid surface $\left(\gamma_{S}(r)=1-\sqrt{1-r^{2}}\right)$, and impose classical no-slip conditions: $\beta_{S}=\beta_{P}=0$. Up to mathematical technicalities,variants of this model could be considered: for instance, one could assume the same type of roughness for the sphere as for the plane.

Note that if we take the parameters $\varepsilon, \beta_{S}$ and $\beta_{P}$ to be zero in the previous models, we are back to the classical situation of a curved and smooth solid falling towards a plane wall. The whole point is to derive the next order terms that are involved in the expression of $\mathcal{F}_{d}$. Note also that, in view of our models, the assumption that the solid translates along $r=0$ is natural. For the first two models, the whole geometry is axisymmetric. For the third model, one can consider rough walls $P^{\varepsilon}$ that are symmetric with respect to $x$ and $y$. In all these configurations, if the initial velocity field of the solid is along $r=0$, both the geometry and the Stokes flow inherit strong symmetry properties, forcing the velocity field of the solid to be along $r=0$ for all time. This 


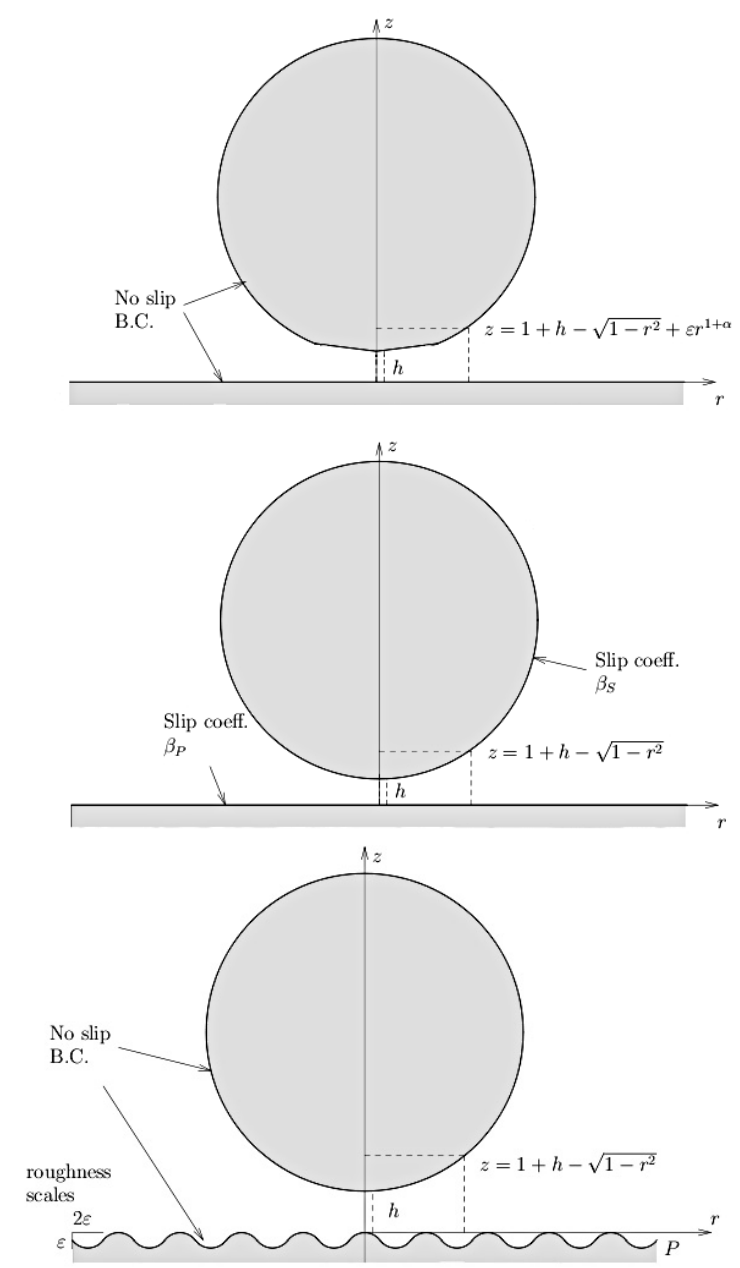

FiguRE 2. Schematic pictures of our three roughness models.

turns to be very convenient for our mathematical analysis. Nevertheless, it could be much weakened, as will be discussed in the conclusion of the paper.

The ambition of this paper is to provide a rigorous and general methodology to derive the drag term $\mathcal{F}_{d}$, in the regime of small distance $h$ between the solid and the wall. This methodology, which relies on the calculus of variations, will be explained in Section 2. Then, in Section 3, it will be applied to our first two models of rough surfaces. In this way, we will extend results from former formal computations, notably those in $[13,18]$. In the last Section 4, we will turn to the third model of a small amplitude and high frequency boundary. This model is of particular interest, as it is connected to the phenomenon of apparent slip, which is a topic of current interest in fluid mechanics, see [17]. We will notably discuss the introduction of an effective slip length as a modelling for hydrophilic rough surfaces.

\section{Methodology FOR DRAG DERIVATION}

We present in this section a general approach to the derivation of the drag force $\mathcal{F}_{d}(t)$ on the solid sphere $S(t)$. We first remark that the geometric configuration at time $t$ is entirely characterized by the distance $h(t)$ 
between the lower tip of the solid and the origin $x=0$. Thus, we can rewrite $S(t)=S_{h(t)}, F(t)=F_{h(t)}$, with the family $\left(S_{h}, F_{h}\right)_{h}$ satisfying

$$
S_{h}=h+S, \quad F_{h}=\left\{\mathrm{x}, \quad \mathrm{x} \notin \overline{S_{h}}, \quad z>\gamma_{P}(x, y)\right\} .
$$

Moreover, considering the linear Stokes equation (1.1) and boundary conditions (1.3)-(1.4), we can write $u(t, \mathrm{x})=\dot{h}(t) u_{h(t)}(\mathrm{x})$ and $p(t, \mathrm{x})=\dot{h}(t) p_{h(t)}(\mathrm{x})$ where $u_{h}, p_{h}$ satisfy the steady problem

$$
-\Delta u_{h}+\nabla p_{h}=0, \quad \operatorname{div} u_{h}=0, \quad \mathrm{x} \in F_{h}
$$

together with the boundary conditions

$$
\left.\left(u_{h}-e_{z}\right) \cdot n\right|_{\partial S_{h}}=0, \quad\left(u_{h}-e_{z}\right) \times\left. n\right|_{\partial S_{h}}=-2 \beta_{S}\left[D\left(u_{h}\right) n\right] \times\left. n\right|_{\partial S_{h}}
$$

and

$$
\left.u_{h} \cdot n\right|_{P}=0, \quad u_{h} \times\left. n\right|_{P}=-2 \beta_{P}\left[D\left(u_{h}\right) n\right] \times\left. n\right|_{\partial P} .
$$

Accordingly, we can write

$$
\mathcal{F}_{d}(t)=\dot{h}(t) \mathcal{F}_{h(t)}, \quad \mathcal{F}_{h}:=\int_{\partial S_{h}}\left(2 D\left(u_{h}\right) n-p_{h} n\right) \mathrm{d} \sigma \cdot e_{z}
$$

The problem is to determine the behaviour of $\mathcal{F}_{h}$ in the limit $h \rightarrow 0$. Our method to address this problem has three main steps:

1. In a first step, we express the drag $\mathcal{F}_{h}$ as the minimum of some energy functional. One can do it using the variational interpretation of (2.1)-(2.3). It allows to identify for all our models of roughness an energy functional $\mathcal{E}_{h}$ and a set of "admissible fields" $\mathcal{A}_{h}$ such that

$$
\mathcal{F}_{h}=\min _{u \in \mathcal{A}_{h}} \mathcal{E}_{h}(u)
$$

The explicit definitions of $\mathcal{E}_{h}$ and $\mathcal{A}_{h}$ will be given at the end of this section;

2. in a second step, we rely on the minimization problem introduced in step 1 to find an accurate lower bound for $\mathcal{F}_{h}$. Namely, we choose some appropriate energy functional $\tilde{\mathcal{E}}_{h} \leq \mathcal{E}_{h}$ and some appropriate set of admissible fields $\tilde{\mathcal{A}}_{h} \supset \mathcal{A}_{h}$ for which we can compute explicitly the minimimum and corresponding minimizer $\tilde{u}$. In this way, we get

$$
\tilde{\mathcal{E}}_{h}(\tilde{u})=\min _{u \in \tilde{\mathcal{A}}_{h}} \tilde{\mathcal{E}}_{h}(u) \leq \min _{u \in \mathcal{A}_{h}} \mathcal{E}_{h}(u)=\mathcal{F}_{h}
$$

which yields a lower bound. Of course, the relaxed functional $\tilde{\mathcal{E}}_{h}$ and admissible set $\tilde{\mathcal{A}}_{h}$ must remain close enough to the original ones, in order for this lower bound to be accurate. We will make them explicit for our various roughness models later on;

3. in a third step, we choose some appropriate field $\check{u} \in \mathcal{A}_{h}$ so that

$$
\mathcal{F}_{h}=\min _{u \in \mathcal{A}_{h}} \mathcal{E}_{h}(u) \leq \mathcal{E}_{h}(\check{u})
$$

provides an accurate upper bound for the drag (that is with the same type of behaviour as the lower one). In many cases, as will be seen later on, the minimizer $\tilde{u} \in \tilde{\mathcal{A}}_{h}$ of the second step generally belongs to the original set of admissible fields $\mathcal{A}_{h}$, or at least can be slightly modified to belong to $\mathcal{A}_{h}$. Thus, one can take in general $\check{u} \approx \tilde{u}$. 
Our goal in the present paper is to apply this methodology to have a better understanding of roughness effects. In this section, we carry out step 1, that is the formulation of the drag in terms of some minimization problem. This step is very general, and independent of the roughness issue. In the next sections, when turning to steps 2 and 3, each roughness model will of course require specific calculations.

To link the drag to an extremum problem, we must distinguish between the case of no slip $\left(\beta_{S}=\beta_{P}=0\right)$ and the case of non-zero slip $\left(\beta_{S}>0, \beta_{P}>0\right)$.

- In the case of no-slip, the divergence free-condition implies

$$
\int_{F_{h}}\left|\nabla u_{h}\right|^{2}=2 \int_{F_{h}}\left|D\left(u_{h}\right)\right|^{2}
$$

Hence, multiplying the Stokes equation $(2.1)$ by $u_{h}$ and integrating over the fluid domain $F_{h}$, we obtain by Stokes formula

$$
2 \int_{F_{h}}\left|D\left(u_{h}\right)\right|^{2}=\int_{\partial S_{h} \cup P}\left(2 D\left(u_{h}\right) n-p_{h} n\right) \cdot u_{h} \mathrm{~d} \sigma=\int_{\partial S_{h}}\left(2 D\left(u_{h}\right) n-p_{h} n\right) \mathrm{d} \sigma \cdot e_{z}=\mathcal{F}_{h} .
$$

Moreover, we know that equation (2.1) (together with the boundary conditions (2.2)-(2.3)) is the Euler equation of a minimization problem. Namely,

$$
\int_{F_{h}}\left|\nabla u_{h}\right|^{2}=\min \left\{\int_{F_{h}}|\nabla u|^{2}, \quad u \in H_{l o c}^{1}\left(F_{h}\right), \quad \nabla \cdot u=0,\left.\quad u\right|_{P}=0,\left.u\right|_{S_{h}}=e_{z}\right\} .
$$

(We remind that the Sobolev space $H_{l o c}^{1}$ is the space of fields $u$ that are locally square integrable, with distributional derivative $\nabla u$ also locally square integrable $)^{3}$.

Indeed, if $u$ has the properties mentioned above, then $u-u_{h}$ is zero along the boundary $\partial S_{h} \cup P$. So, multiplying (1.1) by $u-u_{h}$ and integrating by parts, we end up with

$$
\int_{F_{h}}\left|\nabla u_{h}\right|^{2}=\int_{F_{h}} \nabla u_{h}: \nabla u \leq\left(\int_{F_{h}}\left|\nabla u_{h}\right|^{2}\right)^{1 / 2}\left(\int_{F_{h}}|\nabla u|^{2}\right)^{1 / 2},
$$

using the Cauchy-Schwarz inequality. The characterization of $u_{h}$ follows, and eventually yields that $\mathcal{F}_{h}=$ $\min _{u \in \mathcal{A}_{h}} \mathcal{E}_{h}(u)$, with

$$
\mathcal{E}_{h}(u):=\int_{F_{h}}|\nabla u|^{2}, \quad \mathcal{A}_{h}:=\left\{u \in H_{l o c}^{1}\left(F_{h}\right), \quad \nabla \cdot u=0,\left.\quad u\right|_{P}=0,\left.u\right|_{\partial S_{h}}=e_{z}\right\} .
$$

- In the case of positive slip lengths $\beta_{S}, \beta_{P}$, the minimization problem and the computation that leads to it are slightly different. In particular, the set of admissible fields will be this time

$$
\mathcal{A}_{h}:=\left\{u \in H_{l o c}^{1}\left(F_{h}\right), \quad \nabla \cdot u=0,\left.\quad u \cdot n\right|_{P}=0,\left.\left(u-e_{z}\right) \cdot n\right|_{\partial S_{h}}=0\right\} .
$$

Let $w$ be some divergence-free vector field tangent to the boundary: $w \cdot n=0$ at $\partial S_{h} \cup P$. Multiplying the Stokes equation for $u_{h}$ by $w$, we obtain after integrating by parts:

$$
\int_{F_{h}} 2 D\left(u_{h}\right): D(w)+\frac{1}{\beta_{S}} \int_{\partial S_{h}}\left(\left(u_{h}-e_{z}\right) \times n\right) \cdot(w \times n) \mathrm{d} \sigma+\frac{1}{\beta_{P}} \int_{P}\left(u_{h} \times n\right) \cdot(w \times n) \mathrm{d} \sigma=0 .
$$

In order to recover full gradients instead of symmetric gradients, we proceed as follows. First, we remind the following identity of differential geometry (see for instance [6], Lem. 1, p. 233):

$$
D(\tilde{w}) n \times n=\frac{1}{2} \partial_{n} \tilde{w} \times n+\frac{1}{2} \tilde{w} \times n \text { at } \partial S_{h}
$$

\footnotetext{
${ }^{3}$ As no abstract theory is needed in the remainder of the article, such mathematical details can be skipped without harm.
} 
for any smooth $\tilde{w}$ satisfying $\tilde{w} \cdot n=0$ at $\partial S_{h}$. The last term at the r.h.s is connected to the curvature of $\partial S$, which is simply 1 by our choice of $S$. Similarly,

$$
D(\tilde{w}) n \times n=\frac{1}{2} \partial_{n} \tilde{w} \times n \text { at } P
$$

for any smooth $\tilde{w}$ satisfying $\tilde{w} \cdot n=0$ at $P$. Let now $v$ be some smooth field in $\mathcal{A}_{h}$. Writing

$$
\Delta v=\operatorname{div}(\nabla v), \quad \text { respectively } \Delta v=2 \operatorname{div}(D(v)),
$$

and testing against some smooth $w$ satisfying $w \cdot n=0$ at $\partial S_{h} \cup P$, we obtain

$$
\begin{aligned}
\int_{F_{h}} \Delta v \cdot w & =-\int_{F_{h}} \nabla v: \nabla w+\int_{\partial S_{h}}\left(\partial_{n} v \times n\right) \cdot(w \times n)+\int_{P}\left(\partial_{n} v \times n\right) \cdot(w \times n), \\
& =-\int_{F_{h}} \nabla v: \nabla w+\int_{\partial S_{h}}\left(\partial_{n}\left(v-e_{z}\right) \times n\right) \cdot(w \times n)+\int_{P}\left(\partial_{n} v \times n\right) \cdot(w \times n),
\end{aligned}
$$

respectively

$$
\int_{F_{h}} \Delta v \cdot w=-\int_{F_{h}} 2 D(v): D(w)+\int_{\partial S_{h}}\left(2 D\left(v-e_{z}\right) n \times n\right) \cdot(w \times n)+\int_{P}(2 D(v) n \times n) \cdot(w \times n) .
$$

Combining the last two identities, we get

$$
\begin{aligned}
\int_{F_{h}} 2 D(v): D(w)= & \int_{F_{h}} \nabla v: \nabla w+\int_{\partial S_{h}}\left(2 D\left(v-e_{z}\right) n \times n\right) \cdot(w \times n)+\int_{P}(2 D(v) n \times n) \cdot(w \times n) \\
& -\int_{\partial S_{h}}\left(\partial_{n}\left(v-e_{z}\right) \times n\right) \cdot(w \times n)-\int_{P}\left(\partial_{n} v \times n\right) \cdot(w \times n) .
\end{aligned}
$$

Applying (2.6) (with $\tilde{w}=v-e_{z}$ ) and (2.7) (with $\tilde{w}=v$ ) leads to

$$
\int_{F_{h}} 2 D(v): D(w)=\int_{F_{h}} \nabla v: \nabla w+\int_{\partial S_{h}}\left(\left(v-e_{z}\right) \times n\right) \cdot(w \times n) .
$$

We take $v=u_{h}$ and combine the last equality with (2.5). We obtain

$$
\int_{F_{h}} \nabla u_{h}: \nabla w+\left(\frac{1}{\beta_{S}}+1\right) \int_{\partial S_{h}}\left(\left(u_{h}-e_{z}\right) \times n\right) \cdot(w \times n)+\frac{1}{\beta_{P}} \int_{P}\left(u_{h} \times n\right) \cdot(w \times n)=0 .
$$

Eventually, we take $w=u_{h}-u$ with $u \in \mathcal{A}_{h}$, and substitute into the last equality to obtain

$$
\begin{aligned}
\int_{F_{h}}\left|\nabla u_{h}\right|^{2}+\left(\frac{1}{\beta_{S}}+1\right) & \int_{\partial S_{h}}\left|\left(u_{h}-e_{z}\right) \times n\right|^{2} \mathrm{~d} \sigma+\frac{1}{\beta_{P}} \int_{P}\left|u_{h} \times n\right|^{2} \mathrm{~d} \sigma=\int_{F_{h}} \nabla u_{h}: \nabla u \\
& +\left(\frac{1}{\beta_{S}}+1\right) \int_{\partial S_{h}}\left(\left(u_{h}-e_{z}\right) \times n\right) \cdot\left(\left(u-e_{z}\right) \times n\right) \mathrm{d} \sigma+\frac{1}{\beta_{P}} \int_{P}\left(u_{h} \times n\right) \cdot(u \times n) \mathrm{d} \sigma .
\end{aligned}
$$

Use of the Cauchy-Schwarz inequality and of the Young inequality $\sqrt{a b} \leq \frac{1}{2}(a+b)$ leaves us with

$$
\begin{aligned}
\int_{F_{h}}\left|\nabla u_{h}\right|^{2}+\left(\frac{1}{\beta_{S}}+1\right) \int_{\partial S_{h}} \mid\left(u_{h}\right. & \left.-e_{z}\right) \times\left. n\right|^{2} \mathrm{~d} \sigma+\frac{1}{\beta_{P}} \int_{P}\left|u_{h} \times n\right|^{2} \mathrm{~d} \sigma \\
& \leq \int_{F_{h}}|\nabla u|^{2}+\left(\frac{1}{\beta_{S}}+1\right) \int_{\partial S_{h}}\left|\left(u-e_{z}\right) \times n\right|^{2} \mathrm{~d} \sigma+\frac{1}{\beta_{P}} \int_{P}|u \times n|^{2} \mathrm{~d} \sigma
\end{aligned}
$$


Thus, we have this time that $\mathcal{F}_{h}=\min _{u \in \mathcal{A}_{h}} \mathcal{E}_{h}(u)$, with

$$
\begin{aligned}
\mathcal{E}_{h}(u) & :=\int_{F_{h}}|\nabla u|^{2}+\left(\frac{1}{\beta_{S}}+1\right) \int_{\partial S_{h}}\left|\left(u-e_{z}\right) \times n\right|^{2}+\frac{1}{\beta_{P}} \int_{P}|u \times n|^{2}, \\
\mathcal{A}_{h} & :=\left\{u \in H_{l o c}^{1}\left(F_{h}\right), \quad \nabla \cdot u=0,\left.\quad u \cdot n\right|_{P}=0,\left.\left(u-e_{z}\right) \cdot n\right|_{\partial S_{h}}=0\right\} .
\end{aligned}
$$

We note that contrary to the no-slip case, only the impermeability condition is included in the definition of the space $\mathcal{A}_{h}$. It can be shown that the Euler equation for the latter minimizing problem includes the boundary conditions (2.2)-(2.3) on the tangential part of the velocity-field by standard integration by parts as in the noslip case. For brevity, we shall replace the coefficient $1 / \beta_{S}+1$ by $1 / \beta_{S}$ in what follows. This means that we shall include curvature effects in the slip coefficient. The characterization of the drag through energy functionals (2.4) and (2.8) will be applied to our first two roughness models in the next section.

\section{Application to various roughness models}

In this section, we detail the steps 2 and 3 of our methodology, both in the case of a non-smooth boundary (model 1) and in the case of slip boundary conditions (model 2).

\subsection{The case of non-smooth solids}

As emphasized in the introduction, we consider here the case of an axisymmetric solid $S$, whose boundary is described near its lower tip by

$$
\gamma_{S}(r)=1-\sqrt{1-r^{2}}+\varepsilon r^{1+\alpha}, \quad \alpha \in[0,1], \quad r \leq r_{0}
$$

The wall is flat, and no slip conditions are imposed at all boundaries. The drag is given by

$$
\mathcal{F}_{h}=\min _{u \in \mathcal{A}_{h}} \mathcal{E}_{h}(u),
$$

with the energy $\mathcal{E}_{h}$ and the set of admissible fields $\mathcal{A}_{h}$ given in (2.4).

As the fluid domain $F_{h}$ is invariant by rotations around $e_{z}$, much can be said about the minimizer $u=u_{h}$. Indeed, for any rotation $R_{\theta}$ around $e_{z}, R_{\theta} u_{h} R_{-\theta}$ still belongs to $\mathcal{A}_{h}$, and has the same energy as $u_{h}$. Uniqueness of this minimizer yields

$$
R_{\theta} u_{h}\left(R_{-\theta} \mathrm{x}\right)=u_{h}(\mathrm{x}), \quad \forall \mathrm{x} \in F_{h} .
$$

This means that $u_{h}$ has the following structure:

$$
u_{h}=u_{h, r}(r, z) e_{r}+u_{h, \theta}(r, z) e_{\theta}+u_{h, z}(r, z) e_{z},
$$

where $(r, \theta, z)$, resp. $\left(e_{r}, e_{\theta}, e_{z}\right)$ are the cylindrical coordinates, resp. the cylindrical vector basis. One then remarks that $v_{h}=u_{h, r}(r, z) e_{r}+u_{h, z}(r, z) e_{z}$ still belongs to $\mathcal{A}_{h}$, with $\mathcal{E}_{h}\left(v_{h}\right) \leq \mathcal{E}_{h}\left(u_{h}\right)$. Again, by uniqueness of the minimizer, we get $u_{h}=v_{h}$ and $u_{h, \theta}=0$. Thus, the divergence free condition resumes to

$$
\frac{1}{r} \partial_{r}\left(r u_{h, r}\right)+\partial_{z} u_{h, z}=0 .
$$

Together with the boundary condition $u_{h, z}(r, 0)=0$, it leads to

$$
u_{h}=-\partial_{z} \phi e_{r}+\frac{1}{r} \partial_{r}(r \phi) e_{z}
$$

with streamfunction $\phi(r, z):=-\int_{0}^{z} u_{h, r}\left(r, z^{\prime}\right) \mathrm{d} z^{\prime}$. The boundary conditions on $\phi$ are

$$
\left.\partial_{z} \phi\right|_{\partial S}=\left.\partial_{z} \phi\right|_{P}=0,\left.\quad \partial_{r}(r \phi)\right|_{\partial S}=r,\left.\quad \phi\right|_{P}=0 .
$$


Thus, we can without restriction include these last conditions in the set of admissible fields: instead of the original definition in (2.8), we take

$$
\mathcal{A}_{h}:=\left\{u \in H_{l o c}^{1}\left(F_{h}\right), \quad u=-\partial_{z} \phi e_{r}+\frac{1}{r} \partial_{r}(r \phi) e_{z} \text { for some } \phi \text { satisfying (3.3) }\right\} .
$$

We quote that the boundary conditions on $\phi$ at $\partial S$ yield

$$
\partial_{z} \phi\left(r, h+\gamma_{S}(r)\right)=0, \quad \partial_{r}(r \phi)\left(r, h+\gamma_{S}(r)\right)=r, \quad r<r_{0} .
$$

They imply in turn that $\phi\left(r, h+\gamma_{S}(r)\right)=\frac{r}{2}+\frac{c}{r}$ for some constant $c$. As $\phi(r, z)=-\int_{0}^{z} u_{r}\left(r, z^{\prime}\right) \mathrm{d} z^{\prime}$ is regular enough near $r=0$, we deduce $c=0$. Eventually

$$
\begin{array}{lrl}
\partial_{z} \phi\left(r, h+\gamma_{S}(r)\right)=0, & \phi\left(r, h+\gamma_{S}(r)\right)=\frac{r}{2}, & r<r_{0}, \\
\partial_{z} \phi(r, 0)=0, & \phi(r, 0)=0, & r<r_{0} .
\end{array}
$$

From there, we obtain an accurate lower bound as follows. Noticing that

$$
|\nabla u|^{2}=\left|\partial_{r z} \phi\right|^{2}+\left|\partial_{z} \phi / r\right|^{2}+\left|\partial_{z z} \phi\right|^{2}+\left|\partial_{r}\left[\partial_{r}(r \phi) / r\right]\right|^{2}+\left|\partial_{r z}(r \phi) / r\right|^{2} .
$$

we anticipate that in the limit of small $h$, most of the energy $\mathcal{E}_{h}$ will come from a neighborhood of the lower tip of the sphere

$$
F_{h}^{0}:=\left\{r<r_{0}, 0<z<h+\gamma_{S}(r)\right\},
$$

and will be due to the $z$ derivatives of the stream function $\phi$. Accordingly, we introduce the following relaxed minimizing set and energy functional:

$$
\begin{aligned}
& \tilde{\mathcal{A}}_{h}:=\left\{u \in H^{1}\left(F_{h}^{0}\right), \quad u=-\partial_{z} \phi e_{r}+\frac{1}{r} \partial_{r}(r \phi) e_{z} \text { for some } \phi \text { satisfying (3.4) }\right\}, \\
& \tilde{\mathcal{E}}_{h}:=\int_{F_{h}^{0}}\left|\partial_{z} u_{r}\right|^{2}=\int_{F_{h}^{0}}\left|\partial_{z}^{2} \phi\right|^{2} .
\end{aligned}
$$

From the Euler equation $\partial_{z}^{4} \phi=0$ and the boundary conditions (3.4), it follows easily that the latter minimum is realized with

$$
\tilde{\phi}_{h}(r, z)=\frac{r}{2} \Phi\left(\frac{z}{h+\gamma_{S}(r)}\right), \quad \text { where } \quad \Phi(t)=t^{2}(3-2 t), \quad \forall t \in[0,1]
$$

and has for value:

$$
\tilde{\mathcal{F}}_{h}=6 \pi \int_{0}^{r_{0}} \frac{r^{3} \mathrm{~d} r}{\left(h+\gamma_{S}(r)\right)^{3}} .
$$

We emphasize that this formula is general for no-slip boundary conditions. It does not require any special assumption on the solid surface. In the case $\gamma_{S}(r)=1-\sqrt{1-r^{2}}+\varepsilon r^{1+\alpha}$, our lower bound satisfies

$$
\begin{aligned}
\tilde{\mathcal{F}}_{h} & =6 \pi \int_{0}^{r_{0}} \frac{r^{3} \mathrm{~d} r}{\left(h+\frac{r^{2}}{2}+\varepsilon r^{1+\alpha}+O\left(r^{4}\right)\right)^{3}} \\
& =\frac{6 \pi}{h} \mathcal{I}\left(\varepsilon h^{\frac{\alpha-1}{2}}\right)+O\left(\mathcal{J}\left(\varepsilon h^{\frac{\alpha-1}{2}}, h\right)\right)+O(1)
\end{aligned}
$$

where

$$
\mathcal{I}(\beta):=\int_{0}^{\infty} \frac{s^{3} \mathrm{~d} s}{\left(1+\frac{s^{2}}{2}+\beta s^{1+\alpha}\right)^{3}}, \quad \mathcal{J}(\beta, h):=\int_{0}^{r_{0} / \sqrt{h}} \frac{s^{7} \mathrm{~d} s}{\left(1+\frac{s^{2}}{2}+\beta s^{1+\alpha}\right)^{4}}
$$


The computation of the asymptotic behaviours of $\mathcal{I}$ and $\mathcal{J}$ is detailed in Appendix A. It yields the following results:

- when $\beta \ll 1$, we obtain:

$$
\mathcal{I}(\beta)=\frac{1}{1+\lambda_{\alpha} \beta}+O\left(\beta^{2}\right), \quad \mathcal{J}(\beta, h)=O(|\ln (h)|)
$$

with an explicit constant $\lambda_{\alpha}$ given in the appendix;

- when $\beta \gg 1$, we have:

$$
\mathcal{I}(\beta)= \begin{cases}\mu_{\alpha} \beta^{-\frac{4}{1+\alpha}}+O\left(\frac{1}{\beta^{3}}\right), & \text { for } \alpha>\frac{1}{3}, \\ \frac{9}{4} \frac{\ln (\beta)}{\beta^{3}}+O\left(\frac{1}{\beta^{3}}\right), & \text { for } \alpha=\frac{1}{3}, \\ \mu_{\alpha} \beta^{-\frac{2}{1-\alpha}}+O\left(\frac{1}{\beta^{3}}\right), & \text { for } \alpha<\frac{1}{3},\end{cases}
$$

where the value of $\mu_{\alpha}$ is also provided in the appendix. As regards the remainder, we have the following bound:

$$
\mathcal{J}(\beta, h)=O\left(\left|\ln (\beta)+\frac{1-\alpha}{2} \ln (h)\right|\right) .
$$

Back to the drag force, (3.9) and (3.10) yield the following lower bound: for $\beta=\varepsilon h^{\frac{\alpha-1}{2}} \ll 1$

$$
\tilde{\mathcal{F}}_{h}=\frac{6 \pi}{h+\lambda_{\alpha} \varepsilon h^{\frac{\alpha+1}{2}}}(1+O(\beta))+O(|\ln (h)|)
$$

and for $\beta=\varepsilon h^{\frac{\alpha-1}{2}} \gg 1$

$$
\tilde{\mathcal{F}}_{h}= \begin{cases}\frac{6 \pi \mu_{\alpha}}{\varepsilon^{\frac{4}{1+\alpha}} h^{\frac{3 \alpha-1}{\alpha+1}}}\left(1+\beta^{\frac{1-3 \alpha}{1+\alpha}}\right)+O(|\ln (h)|), & \text { for } \alpha>\frac{1}{3} \\ \frac{9 \pi|\ln (h)|}{2 \varepsilon^{3}}+O\left(\frac{|\ln (\varepsilon)|}{\varepsilon^{3}}\right), & \text { for } \alpha=\frac{1}{3} \\ \frac{6 \pi \mu_{\alpha}}{\varepsilon^{\frac{2}{1-\alpha}}}\left(1+\beta^{\frac{3 \alpha-1}{1+\alpha}}\right)+O(|\ln (\varepsilon)|), & \text { for } \alpha<\frac{1}{3}\end{cases}
$$

Note that the expression given in the case $\alpha<1 / 3$ only matters when $\varepsilon \ll 1$ (otherwise, one can just retain that $\left.\tilde{\mathcal{F}}_{h}=O(1)\right)$.

This concludes our study of a lower bound for the drag. Such bound is accurate, as we can with minor modifications obtain a similar upper bound. Indeed, for $h$ small enough, it is possible to construct a regular stream function $\check{\phi}_{h}=\check{\phi}_{h}(r, z)$ defined on $F_{h}$, equalling $\tilde{\phi}_{h}$ say on $F_{h}^{0} \cap\left\{r<r_{0} / 2\right\}$, such that

$$
\check{u}_{h}=\nabla \times\left(\check{\phi}_{h} e_{\theta}\right) \in \mathcal{A}_{h},
$$

and such that

$$
\int_{F_{h}}\left|\partial_{z}^{2} \check{\phi}_{h}\right|^{2}=\int_{F_{h}^{0}}\left|\partial_{z}^{2} \tilde{\phi}_{h}\right|^{2}+O(1) \text {, uniformly in } h \text { and } \varepsilon \text {. }
$$

The construction of such $\check{\phi}_{h}$ follows closely the one in article [11], Section 4.1, performed in the 2d case. The idea is to introduce

- a truncation function $\chi=\chi(r, z)$ that satisfies

$$
\chi=1 \text { for } 0 \leq r \leq \frac{r_{0}}{2}, 0 \leq z \leq \frac{r_{0}}{2}, \quad \chi=0 \text { for } r>r_{0}, z>r_{0} ;
$$


- a regular fonction $\psi$ which satisfies

$$
\begin{aligned}
& \psi=\frac{r}{2} \text { in a small neighborhood of } S, \\
& \psi=0 \text { in a small neighborhood of } P \cap\left\{r \geq \frac{r_{0}}{2}\right\} .
\end{aligned}
$$

Then, the function $\check{\phi}_{h}$, which equals 1 on $S_{h}$ and is given by

$$
\check{\phi}_{h}(r, z)=(1-\chi(r, z)) \psi(r, z-h)+\chi(r, z) \tilde{\phi}_{h}(r, z) \text { in } F_{h}
$$

satisfies all requirements (still for $h$ small enough). We quote that the remainder term in (3.14) is uniformly bounded, because no singularity is created outside of the contact zone (that is outside a vicinity of $r=0$ ). Eventually, we have:

$$
\begin{aligned}
\mathcal{F}_{h} & \leq \mathcal{E}_{h}\left(\check{u}_{h}\right) \\
& =\tilde{\mathcal{F}}_{h}+\int_{F_{h}}\left[\left|\partial_{r z} \check{\phi}_{h}\right|^{2}+\left|\partial_{z} \check{\phi}_{h} / r\right|^{2}+\left|\partial_{r}\left[\partial_{r}\left(r \check{\phi}_{h}\right) / r\right]\right|^{2}+\left|\partial_{r z}\left(r \check{\phi}_{h}\right) / r\right|^{2}\right]+O(1) \\
& =\tilde{\mathcal{F}}_{h}+\int_{F_{h}^{0}}\left[\left|\partial_{r z} \tilde{\phi}_{h}\right|^{2}+\left|\partial_{z} \tilde{\phi}_{h} / r\right|^{2}+\left|\partial_{r}\left[\partial_{r}\left(r \tilde{\phi}_{h}\right) / r\right]\right|^{2}+\left|\partial_{r z}\left(r \tilde{\phi}_{h}\right) / r\right|^{2}\right]+O(1) .
\end{aligned}
$$

The computation of the integral terms at the r.h.s. follows the lines of Appendix A. It yields some $O(\min (|\ln (h)|,|\ln (\varepsilon)|))$ error term. The main reason for these integrals to be lower order terms is that in the "curved" contact zone, the typical lengthscales in $z$ and $r$ are respectively $h$ and $\sqrt{h}$, so that $z$-derivatives are more singular than $r$-derivatives. Eventually,

$$
\mathcal{F}_{h}=\tilde{\mathcal{F}}_{h}+O(\min (|\ln (\varepsilon)|,|\ln (h)|)) .
$$

We stress that this modelling of the roughness solves the famous no-collision paradox discussed in the introduction. Indeed, as $h$ goes to zero for a given $\varepsilon, \beta$ goes to infinity and the roughness effect yields a drag force $\mathcal{F}_{h}$ which is always bounded by $c_{\varepsilon} h^{-\gamma}$ for some $\gamma<1$. In particular, it is weaker than in the smooth case. Back to the o.d.e.

$$
\ddot{h}+\dot{h} \mathcal{F}_{h}=0
$$

which governs the solid dynamics, integration from time 0 to time $t$ leads to

$$
\dot{h}(t)=\dot{h}(0)+\int_{h(t)}^{h(0)} \mathcal{F}_{h^{\prime}} \mathrm{d} h^{\prime} .
$$

For an initial speed $\dot{h}(0)$ negative enough, $\dot{h}(t)$ remains uniformly negative, because the integral at the r.h.s. is $O\left(h(t)^{1-\gamma}+h(0)^{1-\gamma}\right)=O(1)$. This makes $h$ to cancel in finite time.

\subsection{The case of slip boundary conditions}

We turn in this paragraph to our second model, in which roughness is involved through slip coefficients. We want to have a close approximation of $\mathcal{F}_{h}=\min _{\mathcal{A}_{h}} \mathcal{E}_{h}$, where this time $\mathcal{A}_{h}$ and $\mathcal{E}_{h}$ are defined in (2.8). We still have a rotational invariance in this case, so that we can again reduce $\mathcal{A}_{h}$ by restricting to velocity fields of the type

$$
u=-\partial_{z} \phi e_{r}+\frac{1}{r} \partial_{r}[r \phi] e_{z} .
$$

The impermeability condition at $P$ yields again

$$
\phi(r, 0)=0, \quad \forall r \in\left(0, r_{0}\right) .
$$


As regards $\partial S$, we have for all $r \in\left(0, r_{0}\right)$

$$
\begin{aligned}
e_{z} \cdot n & =\frac{1}{\sqrt{1+\left|\gamma_{S}^{\prime}(r)\right|^{2}}}, \\
u \cdot n & =\frac{1}{\sqrt{1+\left|\gamma_{S}^{\prime}(r)\right|^{2}}}\left(\gamma_{S}^{\prime}(r) \partial_{z} \phi\left(r, h+\gamma_{S}(r)\right)+\frac{1}{r} \partial_{r}(r \phi)\left(s, h+\gamma_{S}(r)\right)\right), \\
& =\frac{1}{\sqrt{1+\left|\gamma_{S}^{\prime}(r)\right|^{2}}} \frac{1}{r} \frac{\mathrm{d}}{\mathrm{d} r}\left[r \phi\left(r, h+\gamma_{S}(r)\right)\right],
\end{aligned}
$$

so that the impermeability condition leads to

$$
\phi\left(r, h+\gamma_{S}(r)\right)=\frac{r}{2}, \quad \forall r \in\left(0, r_{0}\right) .
$$

Accordingly, we introduce the relaxed set

$$
\tilde{\mathcal{A}}_{h}:=\left\{u \in H^{1}\left(F_{h}^{0}\right), \quad u=-\partial_{z} \phi e_{r}+\frac{1}{r} \partial_{r}[r \phi] e_{z}, \phi \text { satisfying (3.15) and (3.17) }\right\},
$$

with $F_{h}^{0}$ defined in the previous section. We then need to define the relaxed energy $\tilde{\mathcal{E}}_{h}$. As in the previous section, we shall keep only $\partial_{z z} \phi$ in the gradient terms. But we shall not change the boundary integrals involved in (2.8). Therefore, we compute:

- on $P, u \times n=\partial_{z} \phi e_{\theta}$;

- on $\partial S \cap\left\{r<r_{0}\right\}$, because of (3.16)-(3.17), $\left(u-e_{z}\right) \times n=\sqrt{1+\left|\gamma_{S}^{\prime}(r)\right|^{2}} \partial_{z} \phi e_{\theta}$.

Hence, we introduce the approximate energy

$$
\tilde{\mathcal{E}}_{h}:=\int_{F_{h}^{0} \cap\left\{r<r_{0}\right\}}\left|\partial_{z z} \phi(r, z)\right|^{2} r \mathrm{~d} r \mathrm{~d} \theta \mathrm{d} z+2 \pi \int_{0}^{r_{0}}\left[\frac{\left(1+\left|\gamma_{S}^{\prime}(r)\right|^{2}\right)^{\frac{3}{2}}}{\beta_{S}}\left|\partial_{z} \phi\left(r, h+\gamma_{S}(r)\right)\right|^{2}+\frac{1}{\beta_{P}}\left|\partial_{z} \phi(r, 0)\right|^{2}\right] r \mathrm{~d} r .
$$

The corresponding mimimization problem is easy, because it amounts to find, for each value of $r<r_{0}$, the minimizer of the functional

$$
\tilde{\mathcal{E}}_{h}(r)=\int_{0}^{h+\gamma_{S}(r)}\left|\phi_{r}^{\prime \prime}(z)\right|^{2} \mathrm{~d} z+\left[\frac{\left(1+\left|\gamma_{S}^{\prime}(r)\right|^{2}\right)^{\frac{3}{2}}}{\beta_{S}}\left|\phi_{r}^{\prime}\left(h+\gamma_{S}(r)\right)\right|^{2}+\frac{1}{\beta_{P}}\left|\phi_{r}^{\prime}(0)\right|^{2}\right]
$$

over functions $\phi_{r}=\phi_{r}(z)$ satisfying the inhomogeneous Dirichlet conditions

$$
\phi_{r}(0)=0, \quad \phi_{r}\left(h+\gamma_{S}(r)\right)=\frac{r}{2} .
$$

This is a one-dimensional minimization problem, with Euler equation $\phi_{r}^{(4)}=0$, endowed with above Dirichlet conditions, plus Robin type condition on $\phi_{r}^{\prime}$ :

$$
\begin{aligned}
& \phi_{r}^{\prime \prime}\left(h+\gamma_{S}(r)\right)+\frac{\left(1+\left|\gamma_{S}^{\prime}(r)\right|^{2}\right)^{\frac{3}{2}}}{\beta_{S}} \phi_{r}^{\prime}\left(h+\gamma_{S}(r)\right)=0, \\
& \phi_{r}^{\prime \prime}(0)-\frac{1}{\beta_{P}} \phi_{r}^{\prime}(0)=0 .
\end{aligned}
$$

After a few computations, the minimum of $\tilde{\mathcal{E}}_{h}$ is obtained for

$$
\tilde{\phi}_{h}(r, z)=\frac{r}{2} \Phi\left(r, \frac{z}{h+\gamma_{S}(r)}\right),
$$


where $\Phi(r, t)$ is the polynomial of degree 3 in $t$ given by

$$
\begin{aligned}
\Phi(r, t):= & -\frac{2\left(\alpha_{S}+\alpha_{S} \alpha_{P}+\alpha_{P}\right)}{12+4\left(\alpha_{S}+\alpha_{P}\right)+\alpha_{P} \alpha_{S}} t^{3}+\frac{3\left(2+\alpha_{S}\right) \alpha_{P}}{12+4\left(\alpha_{S}+\alpha_{P}\right)+\alpha_{P} \alpha_{S}} t^{2} \\
& +\frac{6\left(2+\alpha_{S}\right)}{12+4\left(\alpha_{S}+\alpha_{P}\right)+\alpha_{P} \alpha_{S}} t
\end{aligned}
$$

where:

$$
\alpha_{S}=\alpha_{S}(r):=\frac{\left(1+\left|\gamma_{S}^{\prime}(r)\right|^{2}\right)^{\frac{3}{2}}\left(h+\gamma_{S}(r)\right)}{\beta_{S}}, \quad \alpha_{P}=\alpha_{P}(r):=\frac{\left(h+\gamma_{S}(r)\right)}{\beta_{P}} .
$$

Note that the coefficients of $\Phi$ are uniformly bounded in $\alpha_{S}, \alpha_{P}$, that is in $r<r_{0}, \beta_{P}, \beta_{S}, h$. In the limiting case $\beta_{S}=\beta_{P}=0$ (no-slip limit), we obtain formally $\Phi(t)=-2 t^{3}+3 t^{2}$, in agreement with the computations of the previous section.

We now turn to the lower bound

$$
\begin{aligned}
\tilde{\mathcal{F}}_{h} & =\min _{\tilde{\mathcal{A}}_{h}} \tilde{\mathcal{E}}_{h} \\
& =2 \pi \int_{0}^{r_{0}}\left[\int_{0}^{1}\left|\partial_{t t} \Phi(r, s)\right|^{2} \mathrm{~d} s+\alpha_{S}\left|\partial_{t} \Phi(r, 1)\right|^{2}+\alpha_{P}\left|\partial_{t} \Phi(r, 0)\right|^{2}\right] \frac{r^{3} \mathrm{~d} r}{\left(h+\gamma_{S}(r)\right)^{3}} .
\end{aligned}
$$

We make the last integral more explicit by replacing $\Phi$ by its value. We obtain

$$
\tilde{\mathcal{F}}_{h}=\frac{\pi}{2} \int_{0}^{r_{0}}\left(I_{1}(r)+I_{2}(r)\right) \frac{r^{3} \mathrm{~d} r}{\left(h+\gamma_{S}(r)\right)^{3}}
$$

where the integrands $I_{1}$ and $I_{2}$ are given by

$$
\begin{aligned}
I_{1} & :=\frac{12\left(\alpha_{S}^{2} \alpha_{P}^{2}+5\left(\alpha_{S}^{2} \alpha_{P}+\alpha_{P}^{2} \alpha_{S}\right)+4\left(\alpha_{S}^{2}+\alpha_{P}^{2}\right)+20 \alpha_{S} \alpha_{P}\right)}{\left(12+4\left(\alpha_{S}+\alpha_{P}\right)+\alpha_{S} \alpha_{P}\right)^{2}} \\
I_{2} & :=\frac{144\left(\alpha_{S}+\alpha_{P}\right)}{\left(12+4\left(\alpha_{S}+\alpha_{P}\right)+\alpha_{S} \alpha_{P}\right)^{2}} .
\end{aligned}
$$

Note that $I_{1}$ and $I_{2}$ are uniformly bounded in $\alpha_{S}, \alpha_{P}$, that is in $r<r_{0}, \beta_{P}, \beta_{S}, h$. Thus, expanding $\gamma_{S}$, we obtain:

$$
\begin{aligned}
\tilde{\mathcal{F}}_{h} & =\frac{\pi}{2} \int_{0}^{r_{0}}\left(I_{1}(r)+I_{2}(r)\right) \frac{r^{3} \mathrm{~d} r}{\left(h+\frac{r^{2}}{2}\right)^{3}}+O(\mathcal{J}(0, h)) \\
& =\frac{\pi}{2} \int_{0}^{r_{0}}\left(I_{1}(r)+I_{2}(r)\right) \frac{r^{3} \mathrm{~d} r}{\left(h+\frac{r^{2}}{2}\right)^{3}}+O(|\ln (h)|)
\end{aligned}
$$

where $\mathcal{J}(0, h)$ was introduced in $(3.8)$ and shown to be $O(|\ln (h)|)$. We must now distinguish between two cases, depending on the behaviour of $h / \beta_{S}$ and $h / \beta_{P}$ :

1. either $h / \beta_{S}$ or $h / \beta_{P}$ is of order 1 or larger. Then, either $\alpha_{S}$ or $\alpha_{P}$ is of order 1 or larger. It follows that

$$
c \leq I_{1}(r)+I_{2}(r) \leq C,
$$

for all $r<r_{0}$, where the constants $c, C$ are uniform with respect to all parameters. We then deduce from (3.19) that

$$
\frac{c^{\prime}}{h} \leq \tilde{\mathcal{F}}_{h} \leq \frac{C^{\prime}}{h} .
$$

Note that in the limiting case $\beta_{S}=\beta_{P}=0$ (no-slip limit), we obtain formally:

$$
\alpha_{S}=\alpha_{P}=+\infty, \quad I_{1}=12, \quad I_{2}=0, \quad \tilde{\mathcal{F}}_{h}=\frac{6 \pi}{h}
$$


recovering the classical result. We also emphasize that the regime considered here includes the case where one of the slip coefficients is zero. In particular, the drag force is stronger than $c^{\prime} / h$ in such a case, preventing any collision;

2. both $h / \beta_{S}$ and $h / \beta_{P}$ are small. This case requires more care. We first notice that

$$
\alpha_{P}=\frac{1}{\beta_{P}}\left(h+\frac{r^{2}}{2}+O\left(r^{4}\right)\right), \quad \alpha_{S}=\frac{1}{\beta_{S}}\left(h+\frac{r^{2}}{2}+O\left(r^{2}\left(h+r^{2}\right)\right)\right) .
$$

From there, for $r_{0}$ and $h$ small enough, we get

$$
c J_{1}(r) \leq I_{1}(r) \leq C J_{1}(r), \quad J_{1}(r):=\left(\frac{a_{P}}{1+a_{P}}+\frac{a_{S}}{1+a_{S}}\right)^{2}
$$

where $c, C>0$ and

$$
a_{P}(r):=\frac{1}{\beta_{P}}\left(h+\frac{r^{2}}{2}\right), \quad a_{S}(r):=\frac{1}{\beta_{S}}\left(h+\frac{r^{2}}{2}\right) .
$$

Then, with the change of variable $r=\sqrt{h} u$, we write

$$
\int_{0}^{r_{0}} J_{1}(r) \frac{r^{3} \mathrm{~d} r}{\left(h+\frac{r^{2}}{2}\right)^{3}}=\frac{1}{h} \int_{0}^{\frac{1}{2 \sqrt{h}}}\left(\frac{h / \beta_{P}\left(1+u^{2} / 2\right)}{1+h / \beta_{P}\left(1+u^{2} / 2\right)}+\frac{h / \beta_{S}\left(1+u^{2} / 2\right)}{1+h / \beta_{S}\left(1+u^{2} / 2\right)}\right)^{2} \frac{u^{3} \mathrm{~d} u}{\left(1+u^{2} / 2\right)^{3}} .
$$

In the regime of small $h / \beta_{P}$ and $h / \beta_{S}$, we get that this last integral is $o\left(1 / \beta_{P}+1 / \beta_{S}\right)$. Finally, all of this leads to

$$
\frac{\pi}{2} \int_{0}^{r_{0}} I_{1}(r) \frac{r^{3} \mathrm{~d} r}{\left(h+\frac{r^{2}}{2}\right)^{3}}=o\left(1 / \beta_{P}+1 / \beta_{S}\right)
$$

It now remains to evaluate the contribution of $I_{2}$, which will yield the leading behaviour of $\tilde{\mathcal{F}}_{h}$. The use of (3.20) gives first

$$
\frac{\pi}{2} \int_{0}^{r_{0}} I_{2}(r) \frac{r^{3} \mathrm{~d} r}{\left(h+\frac{r^{2}}{2}\right)^{3}}=\frac{\pi}{2} \int_{0}^{r_{0}} \frac{144\left(a_{S}+a_{P}\right)}{\left(12+4\left(a_{S}+a_{P}\right)+a_{S} a_{P}\right)^{2}} \frac{r^{3} \mathrm{~d} r}{\left(h+\frac{r^{2}}{2}\right)^{3}}+O\left(1 / \beta_{P}+1 / \beta_{S}\right) .
$$

Then, straightforward manipulations show that

$$
\begin{aligned}
\left(\frac{a_{S}}{\left(1+c_{1} a_{S}\right)^{2}}+\frac{a_{P}}{\left(1+c_{1} a_{P}\right)^{2}}\right)+O\left(J_{1}(r)\right) & \leq \frac{144\left(a_{S}+a_{P}\right)}{\left(12+4\left(a_{S}+a_{P}\right)+a_{S} a_{P}\right)^{2}} \\
& \leq\left(\frac{a_{S}}{\left(1+c_{2} a_{S}\right)^{2}}+\frac{a_{P}}{\left(1+c_{2} a_{P}\right)^{2}}\right)+O\left(J_{1}(r)\right)
\end{aligned}
$$


for some $c_{1}, c_{2}>0$. As seen in the treatment of $I_{1}$, the $O\left(J_{1}(r)\right)$ term will only contribute to the drag through a $o\left(1 / \beta_{P}+1 / \beta_{S}\right)$ term. The main contribution of $I_{2}$ to the drag will be governed by

$$
\begin{aligned}
& \frac{\pi}{2} \int_{0}^{r_{0}}\left(\frac{a_{S}}{\left(1+c a_{S}\right)^{2}}+\frac{a_{P}}{\left(1+c a_{P}\right)^{2}}\right) \frac{r^{3} \mathrm{~d} r}{\left(h+\frac{r^{2}}{2}\right)^{3}} \\
& =\frac{\pi}{2 h} \int_{0}^{\frac{1}{2 \sqrt{h}}}\left(\frac{h / \beta_{S}\left(1+u^{2} / 2\right)}{\left(1+c h / \beta_{S}\left(1+u^{2} / 2\right)\right)^{2}}+\frac{h / \beta_{P}\left(1+u^{2} / 2\right)}{\left(1+c h / \beta_{P}\left(1+u^{2} / 2\right)\right)^{2}}\right) \frac{u^{3} \mathrm{~d} u}{\left(1+\frac{u^{2}}{2}\right)^{3}} \\
& =\pi\left\{\frac{1}{\beta_{S}} \int_{1}^{1+\frac{1}{4 h}} \frac{(x-1) \mathrm{d} x}{\left(1+c h / \beta_{S} x\right)^{2} x^{2}}+\frac{1}{\beta_{P}} \int_{1}^{1+\frac{1}{4 h}} \frac{(x-1) \mathrm{d} x}{\left(1+c h / \beta_{P} x\right)^{2} x^{2}}\right\} \\
& =\pi\left\{\frac{1}{\beta_{S}} \int_{1}^{1+\frac{1}{4 h}} \frac{\mathrm{d} x}{\left(1+c h / \beta_{S} x\right)^{2} x}+\frac{1}{\beta_{P}} \int_{1}^{1+\frac{1}{4 h}} \frac{\mathrm{d} x}{\left(1+c h / \beta_{P} x\right)^{2} x}\right\}+O\left(1 / \beta_{P}+1 / \beta_{S}\right) \\
& =\pi\left(\frac{1}{\beta_{S}}+\frac{1}{\beta_{P}}\right)|\ln (h)|+O\left(1 / \beta_{P}+1 / \beta_{S}\right)
\end{aligned}
$$

through standard manipulations. It yields eventually

$$
\frac{\pi}{2} \int_{0}^{r_{0}} I_{2}(r) \frac{r^{3} \mathrm{~d} r}{\left(h+\frac{r^{2}}{2}\right)^{3}}=\pi\left(\frac{1}{\beta_{S}}+\frac{1}{\beta_{P}}\right)|\ln (h)|+O\left(1 / \beta_{P}+1 / \beta_{S}\right) .
$$

Combining (3.19), (3.22) and (3.23), we end up with the following lower bound for the drag:

$$
\tilde{\mathcal{F}}_{h}=\pi\left(\frac{1}{\beta_{S}}+\frac{1}{\beta_{P}}\right)|\ln (h)|+O\left(1 / \beta_{P}+1 / \beta_{S}\right)+O(|\ln (h)|) .
$$

This lower bound is similar to the one derived by Hocking (see [13]).

This concludes our study of a lower bound for the drag. Hence, it remains to obtain a similar upper bound. One could develop the same approach as in the previous section. Namely, one could look for some suitable extension $\tilde{\phi}_{h}$ of $\tilde{\phi}_{h}$, with similar behaviour for its energy. However, due to the elaborate expression (3.18), this would lead to tedious computations. We overcome this technical difficulty as follows:

1. when $h / \beta_{P}$ or $h / \beta_{S}$ is of order 1 or larger, we take $\check{u}_{h}=\nabla \times\left(\check{\phi}_{h} e_{\theta}\right)$, with the "no-slip" streamfunction $\check{\phi}_{h}$ built in the previous section. We obtain with this choice some $O(1 / h)$ upper bound as expected;

2. when $h / \beta_{P}$ and $h / \beta_{S}$ are small, a good way to recover the right asymptotic behaviour is to set $\check{u}_{h}:=$ $\nabla \times\left[\check{\phi}_{h}(r, z) e_{\theta}\right]$ with

in $F_{h}^{0}$, where

$$
\check{\phi}_{h}(r, z):=\frac{r}{2} \Phi\left(r, \frac{z}{h+\gamma_{S}(r)}\right)
$$

$$
\Phi(r, t):=\left(\frac{1}{1+\alpha_{P}}+\frac{1}{1+\alpha_{S}}\right) \frac{t}{2}+\left(\frac{\alpha_{P}}{1+\alpha_{P}}+\frac{\alpha_{S}}{1+\alpha_{S}}\right) \frac{t^{2}}{2} .
$$

We extend then $\check{\phi}_{h}$ to the whole of $F_{h}$ with a stream function having bounded gradients. Calculations similar to the previous ones yield:

$$
\mathcal{E}_{h}\left(\check{u}_{h}\right)=\pi\left(\frac{1}{\beta_{S}}+\frac{1}{\beta_{P}}\right)|\ln (h)|+O\left(1 / \beta_{P}+1 / \beta_{S}\right)+O(|\ln h|)
$$

where we insist that the $O(|\ln h|)$ is uniform with respect to $\beta_{P}$ and $\beta_{S}$. In particular, in the realistic regime of small slip lengths, we obtain the exact same leading behaviour for the lower and upper bounds. For the sake of brevity, we leave the details to the reader. 


\section{The CASE OF A CORRUgated WALL}

In this section, we focus on the third model of roughness described in the introduction, in which the wall has a small amplitude and high frequency oscillation: namely,

$$
z=\varepsilon \gamma\left(\frac{x}{\varepsilon}, \frac{y}{\varepsilon}\right), \quad \varepsilon>0, \quad \gamma=\gamma(X, Y) \text { 1-periodic, } \gamma \leq 0, \max \gamma=\gamma(0,0)=0 .
$$

We remind that the solid is assumed to be smooth, and that no-slip conditions hold both at the solid surface and the wall. We shall pay special attention to the regime $\varepsilon \ll h \ll 1$, that is when the distance between the solid and the wall is much greater than the size of the roughness. We quote that this is the only regime for which this modelling of the roughness is relevant. Indeed, when $h$ becomes comparable to $\varepsilon$, a rescaling in space by a factor $1 / \varepsilon$ brings back to the classical situation of smooth boundaries. From this point of view, the model we consider in this section is peculiar: it does not allow to conclude anything about the possibility of collisions for a given small roughness size $\varepsilon$. However, we believe that it is still interesting from a purely computational point of view.

Such roughness model with a small parameter is very popular, as it allows for multiscale analysis. This analysis has been notably performed in the context of wall laws. In this context, the idea is to replace the rough boundary by a flat one, and to impose there some good homogenized boundary condition, that expresses the mean effect of roughness. This homogenization problem has been considered by physicists since the early 90 's, through numerics and explicit calculations for special geometries: see for instance [20]. It has been adressed later on in some mathematical works, based on homogenization theory. We refer to $[1,14]$ for periodic patterns of roughness, and to $[3,10]$ for random roughness. The conclusion of these works is that, for small enough $\varepsilon$, one can replace the oscillating boundary by the flat one $\{z=0\}$, and impose there some Navier-type boundary condition:

$$
u_{z}=0, \quad\left(u_{x}, u_{y}\right)=\varepsilon B \partial_{z}\left(u_{x}, u_{y}\right)
$$

for some two by two positive matrix $B$, which is sometimes called the "mobility tensor". There has been a recent interest on qualitative properties of this tensor, for instance for shape optimization in microfluidics, $c f$. [15].

Another frequent idea is that a slip condition amounts to a no-slip condition at a shifted wall. Combining this idea with the previous one, some recent articles have suggested a drag force of the type $\mathcal{F}_{h} \sim \frac{1}{h+\beta \varepsilon}$ for some positive $\beta$ : see $[16,18]$. We will discuss this result in a rigorous manner here.

First, one can use the methodology of Section 2 to derive some lower and upper bounds.

- As regards the lower bound, let us show that

$$
\mathcal{F}_{h} \geq \frac{6 \pi}{h+\lambda \varepsilon}+O(|\ln (h+\lambda \varepsilon)|), \quad \text { for } \lambda:=-\min \gamma>0 .
$$

Indeed, we have

$$
\mathcal{F}_{h}=\min _{u \in \mathcal{A}_{h}} \mathcal{E}_{h}(u)
$$

where $\mathcal{E}_{h}(u)$ and $\mathcal{A}_{h}$ are given by (2.4). Let us now define

$$
P^{\lambda}:=\{z=-\varepsilon \lambda\}, \quad F_{h}^{\lambda}:=\left\{\mathrm{x}, \quad \mathrm{x} \notin \bar{S}_{h}, \quad z>-\varepsilon \lambda\right\} .
$$

Any field $u$ of $\mathcal{A}_{h}$ can be extended by zero below the rough wall so that it can be seen as an element of the larger set

Then, obviously,

$$
\tilde{\mathcal{A}}_{h}:=\left\{u \in H_{l o c}^{1}\left(F_{h}^{\lambda}\right), \quad \nabla \cdot u=0,\left.\quad u\right|_{P^{\lambda}}=0,\left.u\right|_{\partial S_{h}}=e_{z}\right\} .
$$

$$
\mathcal{F}_{h} \geq \min _{u \in \tilde{\mathcal{A}}_{h}} \mathcal{E}_{h}(u)
$$

But the r.h.s of this inequality is exactly the drag force associated to the (smooth) solid $S_{h}$ and the (smooth) plane $P^{\lambda}$. As the distance between the two is $h+\lambda \varepsilon$, we deduce the expected lower bound; 
- with similar arguments, one has the upper bound:

$$
\mathcal{F}_{h} \leq \frac{6 \pi}{h}+O(|\ln (h)|) .
$$

Indeed, let us define this time

$$
P^{0}:=\{z=0\}, \quad F_{h}^{0}:=\left\{\mathrm{x}, \quad \mathrm{x} \notin \bar{S}_{h}, \quad z>0\right\} .
$$

Let $u_{h}^{0}$ be the Stokes flow in the smooth domain $F_{h}^{0}$. As the distance between $S_{h}$ and $P^{0}$ is $h$, the drag force satisfies

$$
\int_{F_{h}^{0}}\left|\nabla u_{h}^{0}\right|^{2}=\frac{6 \pi}{h}+O(|\ln (h)|) .
$$

Now, $u_{h}^{0}$ can be extended by zero below $P^{0}$ and defines in this way an element of $\mathcal{A}_{h}$. In particular, $\mathcal{F}_{h} \leq$ $\int_{F_{h}}\left|\nabla u_{h}^{0}\right|^{2}$.

Hence, our methodology allows to derive quickly the inequalities

$$
\frac{6 \pi}{h+\lambda \varepsilon}+O(|\ln (h+\lambda \varepsilon)|) \leq \mathcal{F}_{h} \leq \frac{6 \pi}{h}+O(|\ln (h)|) .
$$

Interestingly, these bounds are satisfied for any regime of parameters $\varepsilon$ and $h$. In particular, it provides the right asymptotic when $\varepsilon$ and $h$ are of the same order.

Nevertheless, when $\varepsilon \ll h$, it is fair to notice that a multiscale analysis gives a much refined description of the drag. For the sake of completeness, we briefly present it here. It relies on an asymptotic expansion of the Stokes flow $u_{h}=u_{h}^{\varepsilon}$ with respect to $\varepsilon$. This expansion has already been described in close contexts, for instance in [14], and we only recall its main elements. To keep track of the $\varepsilon$ dependency, we write $P^{\varepsilon}$ instead of $P, F_{h}^{\varepsilon}$ instead of $F_{h}$. We denote again $P^{0}$ and $F_{h}^{0}$ their smooth counterparts.

The basic idea is to build an approximate solution $u_{h, \text { app }}^{\varepsilon}(\mathrm{x})$ of $(2.1)-(2.3)$, in the form of an expansion in powers of $\varepsilon$ :

$$
u_{h, a p p}^{\varepsilon}(\mathrm{x})=u_{h}^{0}(\mathrm{x})+\varepsilon\left(u_{h}^{1}(\mathrm{x})+U_{h}^{1}(x, y, \mathrm{x} / \varepsilon)\right)+\ldots+\varepsilon^{N}\left(u_{h}^{N}(\mathrm{x})+U_{h}^{N}(x, y, \mathrm{x} / \varepsilon)\right) .
$$

Each term of this expansion has two parts:

- a regular part $u_{h}^{i}=u_{h}^{i}(\mathrm{x})$ which models the macroscopic variations of the solution;

- a boundary layer correction $U_{h}^{i}=U_{h}^{i}(x, y, \mathrm{X})$, which accounts for the fast variations of the solution near the oscillating boundary. Hence, it depends on the macroscopic variables $x, y$, but also on the microscopic variable $\mathrm{X}=\mathrm{x} / \varepsilon$. It is defined for all

$$
x, y \in \mathbb{R}^{2}, \quad \mathrm{X}=(X, Y, Z) \text { such that } Z>\gamma(X, Y) .
$$

Moreover, $U_{h}^{i}$ is periodic in $X, Y$ (due to the periodicity of the rough bondary $\gamma$ in $X, Y$ ) and satisfies

$$
\lim U_{h}^{i}(x, y, X, Y, Z)=0, \quad \text { as } Z \rightarrow+\infty .
$$

Back to the original variable $\mathrm{x}$, this last condition corresponds to a boundary layer of typical size $\varepsilon$ near the rough wall $P$.

Accordingly, the corresponding pressure field should read

$$
p_{h, a p p}^{\varepsilon}(\mathrm{x})=p_{h}^{0}(\mathrm{x})+P_{h}^{0}(x, y, \mathrm{x} / \varepsilon)+\varepsilon\left(p_{h}^{1}(\mathrm{x})+P_{h}^{1}(x, y, \mathrm{x} / \varepsilon)\right)+\ldots
$$


We remind here briefly how to derive the $O(1)$ and $O(\varepsilon)$ terms, which is enough for our purpose. This kind of derivation (with expansions of boundary layer type) has been performed in various contexts (Laplace, Stokes, Navier-Stokes equations). For more details, one can refer to $[1,3,14]$.

First, if we inject the above expansions in (2.1)-(2.3), and let $Z \rightarrow+\infty$, we obtain by standard manipulations that

$$
-\Delta u_{h}^{0}+\nabla p_{h}^{0}=0 \text { and } \operatorname{div} u_{h}^{0}=0 \text { in } F_{h}^{0},\left.\quad u_{h}^{0}\right|_{\partial S_{h}}=e_{z},\left.\quad u_{h}^{0}\right|_{P^{0}}=0 .
$$

Thus, we recover as expected that the leading term of the expansion is the Stokes flow without roughness. We then extend $u_{h}^{0}$ and $p_{h}^{0}$ by zero below $P^{0}$, so that they are defined over the whole $F_{h}^{\varepsilon}$. Such extensions trivially satisfy the Stokes equation for $z<0$, as well as the no-slip condition at $P^{\varepsilon}$. Moreover, the velocity is continuous across the plane $\{z=0\}$. But there is a $O(1)$ jump in the normal derivative. This explains the introduction of a boundary layer corrector with amplitude $O(\varepsilon)$. Indeed, its gradient has amplitude $O(1)$, and allows to correct this artificial jump.

Let us introduce the following notations:

$$
\begin{aligned}
& V_{h}(x, y, \mathrm{X}):=u_{h}^{1}(x, y, 0)+U_{h}^{1}(x, y, \mathrm{X}), \quad Z>\gamma(X, Y), \\
& P_{h}(x, y, \mathrm{X}):=P_{h}^{0}(x, y, \mathrm{X})-p_{h}^{0}(x, y, 0), \quad Z>0, \\
& P_{h}(x, y, \mathrm{X}):=P_{h}^{0}(x, y, \mathrm{X}), \quad 0>Z>\gamma(X, Y) .
\end{aligned}
$$

Note that, following the expansion (4.1), $u^{0}(x, y, 0)+\varepsilon V_{h}(x, y, \mathrm{x} / \varepsilon)$ should be an approximation of the whole flow in the boundary layer. Plugging (4.1) into the equations, we derive formally the following Stokes system:

$$
\left\{\begin{array}{rlrl}
-\Delta_{\mathrm{X}} V_{h}+\nabla_{\mathrm{X}} P_{h} & =0, & Z>\gamma(X, Y), Z \neq 0, \\
\nabla_{\mathrm{X}} \cdot V_{h}=0, & Z>\gamma(X, Y), Z \neq 0, \\
V_{h}=0, & Z=\gamma(X, Y),
\end{array}\right.
$$

together with the jump conditions

$$
\left.V_{h}\right|_{Z=0^{+}}-\left.V_{h}\right|_{Z=0^{-}}=0,\left.\quad\left(\partial_{Z} V_{h}-P_{h} e_{Z}\right)\right|_{Z=0^{+}}-\left.\left(\partial_{Z} V_{h}-P_{h} e_{Z}\right)\right|_{Z=0^{-}}=-\partial_{z} u_{h}^{0}(x, y, 0) .
$$

Again, we stress that these jump conditions ensure the smoothness of the whole flow across the artificial boundary $\{z=0\}$. Note that by the divergence-free condition $\partial_{z} u_{h, z}^{0}(x, y, 0)=0$, so that only the horizontal components of $\partial_{z} u_{h}^{0}(x, y, 0)$ are non-zero. Let us also point out that the variables $x, y$ are just parameters in the system. In other words, one has

$$
V_{h}(x, y, \mathrm{X})=\mathcal{V}(\mathrm{X}) \partial_{z} u_{h}^{0}(x, y, 0), \quad P_{h}(x, y, \mathrm{X})=\mathcal{P}(\mathrm{X}) \cdot \partial_{z} u_{h}^{0}(x, y, 0)
$$

for some 3 -by-3 matrix function $\mathcal{V}$ and some 3 d vector $\mathcal{P}$ which satisfy the (matricial) Stokes system

$$
\left\{\begin{aligned}
-\Delta_{\mathrm{X}} \mathcal{V}+\nabla_{\mathrm{X}} \mathcal{P}=0, & Z>\gamma(X, Y), \\
\nabla_{\mathrm{X}} \cdot \mathcal{V}=0, & Z>\gamma(X, Y), \\
\mathcal{V}=0, & Z=\gamma(X, Y),
\end{aligned}\right.
$$

together with

$$
\left.\mathcal{V}\right|_{Z=0^{+}}-\left.\mathcal{V}\right|_{Z=0^{-}}=0,\left.\quad\left(\partial_{Z} \mathcal{V}-\mathcal{P} \otimes e_{Z}\right)\right|_{Z=0^{+}}-\left.\left(\partial_{Z} \mathcal{V}-\mathcal{P} \otimes e_{Z}\right)\right|_{Z=0^{-}}=-\left(\begin{array}{ccc}
1 & 0 & 0 \\
0 & 1 & 0 \\
0 & 0 & 0
\end{array}\right)
$$


This system of pde's, depending only on $\mathrm{X}$, with periodic boundary conditions in the horizontal variable $X, Y$, has been extensively studied. We remind the following proposition, extracted from [14]:

Proposition 4.1. The solution $\mathcal{V}$ of system (4.2) converges exponentially at infinity, that is

$$
\left|\mathcal{V}(X, Y, Z)-\mathcal{V}^{\infty}\right| \leq C \mathrm{e}^{-\delta Z}
$$

for some constant 3-by-3 matrix $\mathcal{V}^{\infty}$ and some $\delta>0$. Moreover, $\mathcal{V}^{\infty}$ is of the form

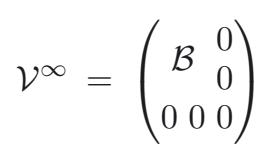

for some symmetric positive definite 2-by-2 matrix $\mathcal{B}$.

The non-zero block $\mathcal{B}$ is sometimes called the mobility tensor, see [15]. We stress that $\mathcal{B}$ is symmetric and definite positive, so diagonalizable in an orthonormal basis with positive eigenvalues. This fact will be used below.

Back to $V_{h}$, we obtain that

$$
\left(V_{h, x}, V_{h, y}\right)(x, y, \mathrm{X}) \rightarrow \mathcal{B} \partial_{z}\left(u_{h, x}^{0}, u_{h, y}^{0}\right)(x, y, 0), \quad V_{h, z}(x, y, \mathrm{X}) \rightarrow 0, \quad \text { as } Z \rightarrow+\infty .
$$

As the boundary layer correction $U_{h}^{1}$ should decay at infinity, we obtain the boundary condition for the macroscopic correction $u_{h}^{1}$ at $P^{0}$. That is

$$
\left(u_{h, x}^{1}, u_{h, y}^{1}\right)=\mathcal{B} \partial_{z}\left(u_{h, x}^{0}, u_{h, y}^{0}\right), \quad u_{h, z}^{1}=0, \quad \text { at } P^{0} .
$$

Together with the Stokes equations

$$
-\Delta u_{h}^{1}+\nabla p_{h}^{1}=0 \text { and } \operatorname{div} u_{h}^{1}=0 \text { in } F_{h}^{0},
$$

and the boundary condition at the solid surface

$$
\left.u_{h}^{1}\right|_{\partial S_{h}}=0
$$

this determines $u_{h}^{1}$, and ends the derivation of the $O(\varepsilon)$ term of the expansion. The next order terms solve the same kind of equations, with inhomogeneous data coming from lower order profile.

In a second step, one can show rigorously that the approximate solution $u_{h, a p p}^{\varepsilon}$ is close to the exact solution $u_{h}^{\varepsilon}$. Indeed, introducing the differences

$$
v:=u_{h, a p p}^{\varepsilon}-u_{h}^{\varepsilon}, \quad \text { and } \quad q:=p_{h, a p p}^{\varepsilon}-p_{h}^{\varepsilon}
$$

leads to

$$
-\Delta v+\nabla q=R_{h}^{\varepsilon}, \quad \operatorname{div} v=r_{h}^{\varepsilon},\left.\quad v\right|_{\partial S_{h}}=\varphi_{h}^{\varepsilon},
$$

with remainder terms $R_{h}^{\varepsilon}, r_{h}^{\varepsilon}$ and $\varphi_{h}^{\varepsilon}$. For instance, the boundary data $\varphi_{h}^{\varepsilon}$ is due to the boundary layer terms $U^{i}(\mathrm{x}, \mathrm{x} / \varepsilon)$, that do not vanish at $\partial S_{h}$. We stress that the assumption $\varepsilon \ll h$ is crucial for these remainders to be small. First, the boundary layer corrections decay exponentially over a typical lengthscale $\varepsilon$. To make it exponentially small at $\partial S_{h}$, one needs $\varepsilon \ll h$. Moreover, all other remainder terms are small with respect to $\varepsilon$, but diverging with respect to $h$. Very roughly, they behave like $O\left((\varepsilon / h)^{N}\right)$ where $N$ is the number of terms in the expansion (4.1). The diverging powers of $h$ come from taking derivatives of the $u_{h}^{i}$, which are singular with respect to $h$. Again, the smallness condition $\varepsilon \ll h$ is necessary. 
From there, as the remainder terms are small, one can through energy estimates deduce the smallness of $v$, that is $u_{h}^{\varepsilon} \approx u_{h, a p p}^{\varepsilon}$. In particular, for $\varepsilon$ small enough compared to $h$, the drag force on $\partial S_{h}$ reads

$$
\mathcal{F}_{h}=\int_{\partial S_{h}}\left(\frac{\partial u_{h}^{\varepsilon}}{\partial n}-p_{h}^{\varepsilon} n\right) \cdot e_{z}=\int_{\partial S_{h}}\left(\frac{\partial\left(u_{h}^{0}+\varepsilon u_{h}^{1}\right)}{\partial n}-\left(p_{h}^{0}+\varepsilon p_{h}^{1}\right) n\right) \cdot e_{z}+o(\varepsilon) .
$$

Moreover, it is easily seen that the fields $\check{u}_{h}^{\varepsilon}:=u_{h}^{0}+\varepsilon u_{h}^{1}, \check{p}_{h}^{\varepsilon}:=p_{h}^{0}+\varepsilon p_{h}^{1}$ satisfy the Stokes equation in $F_{h}^{0}$, together with the boundary conditions

$$
\left.\check{u}_{h}^{\varepsilon}\right|_{\partial S_{h}}=e_{z}, \quad \text { and }\left.\quad \check{u}_{h, z}^{\varepsilon}\right|_{P^{0}}=0,\left.\quad\left(\check{u}_{h, x}^{\varepsilon}, \check{u}_{h, y}^{\varepsilon}\right)\right|_{P^{0}}=\left.\varepsilon \mathcal{B} \partial_{z}\left(u_{h, x}^{0}, u_{h, y}^{0}\right)\right|_{P^{0}} .
$$

By the axisymmetry of $F_{h}^{0}, R_{\theta} u_{h}^{0}=u_{h}^{0} R_{\theta}$ for any horizontal rotation $R_{\theta}$. As $\mathcal{B}$ is symmetric definite positive, this allows us to assume, up to a change of orthonormal basis, that $\mathcal{B}$ is diagonal with positive coefficients $\beta_{x}, \beta_{y}$. Now, there are two ways to make formula (4.4) more explicit.

- On one hand, one can write the latter boundary condition as a slip condition of Navier type:

$$
\left.\left(\check{u}_{h, x}^{\varepsilon}, \check{u}_{h, y}^{\varepsilon}\right)\right|_{P^{0}}=\left.\varepsilon \mathcal{B} \partial_{z}\left(\check{u}_{h, x}^{\varepsilon}, \check{u}_{h, y}^{\varepsilon}\right)\right|_{P^{0}}+o(\varepsilon) .
$$

This is the so-called phenomenon of apparent slip, see [17]. In the isotropic case $\beta:=\beta_{x}=\beta_{y}$, one can use the bounds on the drag force derived in Section 3. In the regime $\varepsilon^{-1} h \gg 1$, this yields:

$$
\frac{c}{h} \leq \mathcal{F}_{h} \leq \frac{C}{h}, \quad c, C>0 ;
$$

- on the other hand, the drag force reads

$$
\mathcal{F}_{h} \approx \int_{\partial S_{h}}\left(\frac{\partial u_{h}^{0}}{\partial n}-p_{h}^{0} n\right) \cdot e_{z}+\varepsilon \int_{\partial S_{h}}\left(\frac{\partial u_{h}^{1}}{\partial n}-p_{h}^{1} n\right) \cdot e_{z} .
$$

Testing the Stokes equation for $u_{h}^{1}$ with $u_{h}^{0}$, we can express the second term at the r.h.s. differently, that is

$$
\mathcal{F}_{h}=\int_{\partial S_{h}}\left(\frac{\partial u_{h}^{0}}{\partial n}-p_{h}^{0} n\right) \cdot e_{z}+\varepsilon \int_{F_{h}^{0}} \nabla u_{h}^{1}: \nabla u_{h}^{0} .
$$

Testing the Stokes equation for $u_{h}^{0}$ with $u_{h}^{1}$, we obtain

$$
\begin{aligned}
\mathcal{F}_{h} & =\int_{\partial S_{h}}\left(\frac{\partial u_{h}^{0}}{\partial n}-p_{h}^{0} n\right) \cdot e_{z}+\varepsilon \int_{P^{0}} u_{h}^{1} \cdot\left(\frac{\partial u_{h}^{0}}{\partial n}-p_{h}^{0} n\right) \\
& =\int_{\partial S_{h}}\left(\frac{\partial u_{h}^{0}}{\partial n}-p_{h}^{0} n\right) \cdot e_{z}-\varepsilon \int_{P^{0}}\left(\beta_{x}\left|\partial_{z} u_{h, x}^{0}\right|^{2}+\beta_{y}\left|\partial_{z} u_{h, y}^{0}\right|^{2}\right)
\end{aligned}
$$

where the last line comes from the boundary conditions (4.3). Using again the symmetry properties of $u_{h}^{0}$, we obtain that

$$
\mathcal{F}_{h} \approx \int_{\partial S_{h}}\left(\frac{\partial u_{h}^{0}}{\partial n}-p_{h}^{0} n\right) \cdot e_{z}-\varepsilon \beta \int_{P^{0}}\left(\left|\partial_{z} u_{h, x}^{0}\right|^{2}+\left|\partial_{z} u_{h, y}^{0}\right|^{2}\right), \quad \beta:=\frac{\beta_{x}+\beta_{y}}{2} .
$$

But this last expression can be seen as the drag force created by a Stokes flow $u_{h}^{\beta}$, between the solid $S_{h}$ and a shifted wall $P_{\beta}:=z=-\varepsilon \beta$. Indeed, following [18], we get

$$
\begin{aligned}
\int_{\partial S_{h}}\left(\frac{\partial u_{h}^{\beta}}{\partial n}-p_{h}^{\beta} n\right) \cdot e_{z} & =\int_{\partial S_{h}}\left(\frac{\partial u_{h}^{\beta}}{\partial n}-p_{h}^{\beta} n\right) \cdot u_{h}^{0} \\
& =\int_{F_{h}^{0}} \nabla u_{h}^{\beta}: \nabla u_{h}^{0} \\
& =\int_{\partial S_{h}^{0}} e_{z} \cdot\left(\frac{\partial u_{h}^{0}}{\partial n}-p_{h}^{0} n\right)+\int_{P^{0}} u_{h}^{\beta} \cdot\left(\frac{\partial u_{h}^{0}}{\partial n}-p_{h}^{0} n\right)
\end{aligned}
$$


Using that

$$
u_{h}^{\beta}(x, y, 0)=u_{h}^{\beta}(x, y,-\varepsilon \beta)+\varepsilon \beta \partial_{z} u^{\beta}(x, y,-\varepsilon \beta)+o(\varepsilon)=\varepsilon \beta \partial_{z} u_{h}^{0}(x, y, 0)+o(\varepsilon)
$$

we recover the same expression as in (4.6). This interpretation of the roughness effect as a shift of the smooth wall yields

$$
\mathcal{F}_{h} \approx \frac{6 \pi}{h+\varepsilon \beta} .
$$

Note that formula (4.5) and (4.7) are coherent, since we are here within the asymptotics $\varepsilon \ll h \ll 1$. As pointed out at the beginning of this paragraph, these formula do not apply to the single limit $h \rightarrow 0$ (for a given $\varepsilon$ ).

\section{FinAl COMMENTS}

Through our variational point of view on the drag problem, we have been able to derive accurate expressions for the drag force, depending on the modelling and amount of roughness at the sphere or at the plane. These expressions are synthetized in Table 1.

The configurations that we have investigated are fairly simple, both with respect to the geometry (axisymmetry assumption, boundary described by a graph) and to the solid dynamics (vertical translation). This simplicity makes the mathematical analysis easier, but is not necessary. Indeed, our derivation of the drag asymptotics relies on "local" arguments. In other words, for a small distance $h$ between the solid and the wall, the singular part of the drag force (obtained through our approximate minimizer $\tilde{u}_{h}$ ) depends only on a small zone between the tip of the solid and the wall (that is $F_{h}^{0}$, see (3.5)). On this basis, we believe that, as long as the characteristics of the solid and wall regions close to contact do not change with time (similar $C^{1, \alpha}$ regularity for the first case, similar slip length for the second case), our expressions for the drag force remain relevant. Nevertheless, the mathematical justification of this claim may become very intricate. Of course, the worst situation is when the solid and wall surfaces have some regions with distinct roughness properties, that alternatively get close to contact.

As regards the third model of roughness, let us stress again that it makes sense only in the regime $\varepsilon \ll h \ll 1$ : indeed, as soon as $h \sim \varepsilon$, there is no separation of scales anymore, and by setting the zoom factor to $1 / \varepsilon$, one is back to the classical situation of smooth solids. One could also wonder if the isotropy assumption that is made on the roughness (with typical size $\varepsilon$ in any direction) is a big restriction. This is not so. Indeed, the isotropic scaling is the richest one (in the sense of asymptotic expansions), as it keeps all terms in the boundary layer equations. All other anisotropic limits can be seen as degenerate subcases of the isotropic one.

Let us finally point out that the present paper is helpful at least with regards to two problems:

- the "no-collision" paradox. We have shown rigorously that accounting for roughness (through a lack of smoothness or a Navier type boundary condition) depletes the singularity of the drag force, resulting in a possible collision in finite time. The main point is that one can solve the no-collision issue within the macroscopic Navier-Stokes model: there is no need to refer to a molecular behaviour;

- the determination of the right hydrodynamic boundary conditions. As mentioned in the introduction, the determination of the right boundary condition at a rough boundary is a topic of active research in microfluidics (see $[16,24])$. One idea to progress on this issue is to make experiments and to measure the drag force exerted on a small sphere close to the rough boundary. Then, one can compare the asymptotics of this measured drag to the asymptotics of theoretical drags issued from different wall laws. By providing an accurate expression for the theoretical drags, our paper can serve as a basis to such investigations. Let us stress that, with the expression of the drag force, one has access to other measurable quantities. For instance, one can compute collision times, by solving the ordinary differential equation

$$
\ddot{h}+\dot{h} \mathcal{F}_{h}=f
$$

where $\mathcal{F}_{h}$ is the normalized drag, and $f$ accounts for other forcings, such as gravity or buoyancy. 


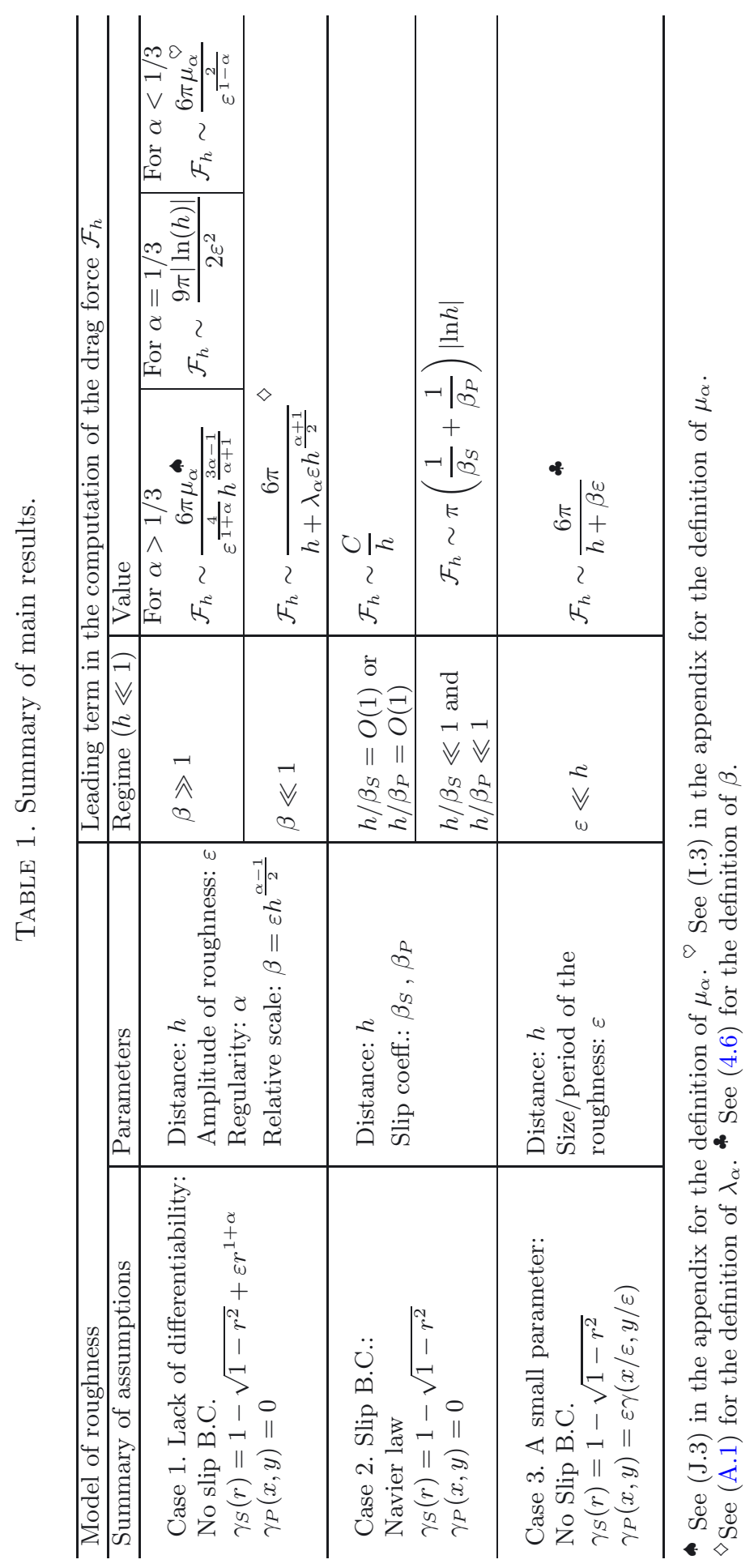




\section{Appendix A. Asymptotics of $\mathcal{I}$ and $\mathcal{J}$}

In this appendix, we detail the computation of $\mathcal{I}(\beta)$ and $\mathcal{J}(\beta, h)$ defined in (3.8) depending on the values of $\beta$.

Case $\beta \ll 1$. When $\beta$ is small, we expand with respect to $\beta$ :

$$
\frac{s^{3}}{\left(1+\frac{s^{2}}{2}+\beta s^{1+\alpha}\right)^{3}}=\frac{s^{3}}{\left(1+\frac{s^{2}}{2}\right)^{3}}-3 \beta \frac{s^{4+\alpha}}{\left(1+\frac{s^{2}}{2}\right)^{4}}+O\left(\beta^{2} \frac{s^{5+2 \alpha}}{\left(1+\frac{s^{2}}{2}\right)^{5}}\right) .
$$

This yields:

$$
\mathcal{I}(\beta)=\int_{0}^{\infty} \frac{s^{3} \mathrm{~d} s}{\left(1+\frac{s^{2}}{2}\right)^{3}}-\beta \int_{0}^{\infty} \frac{3 s^{4+\alpha} \mathrm{d} s}{\left(1+\frac{s^{2}}{2}\right)^{4}}+O\left(\beta^{2}\right)
$$

where routine calculations yield:

$$
\int_{0}^{\infty} \frac{s^{3} \mathrm{~d} s}{\left(1+\frac{s^{2}}{2}\right)^{3}}=1, \quad \int_{0}^{\infty} \frac{3 s^{4+\alpha} \mathrm{d} s}{\left(1+\frac{s^{2}}{2}\right)^{4}}=\frac{2^{\frac{\alpha+1}{2}} \pi(3+\alpha)\left(1-\alpha^{2}\right)}{8 \cos \left(\frac{\pi \alpha}{2}\right)}=: \lambda_{\alpha} .
$$

Replacing in $\mathcal{I}(\beta)$, we obtain,

$$
\mathcal{I}(\beta)=1-\lambda_{\alpha} \beta+O\left(\beta^{2}\right)=\frac{1}{1+\lambda_{\alpha} \beta}+O\left(\beta^{2}\right)
$$

Case $\beta \gg 1$. When $\beta$ is large, we split $\mathcal{I}(\beta)=\mathcal{I}^{0}(\beta)+\mathcal{I}^{\infty}(\beta)$ where:

$$
\mathcal{I}^{0}(\beta)=\int_{0}^{1} \frac{s^{3} \mathrm{~d} s}{\left(1+\frac{s^{2}}{2}+\beta s^{1+\alpha}\right)^{3}}, \quad \mathcal{I}_{\beta}^{\infty}=\int_{1}^{\infty} \frac{s^{3} \mathrm{~d} s}{\left(1+\frac{s^{2}}{2}+\beta s^{1+\alpha}\right)^{3}} .
$$

To compute $\mathcal{I}^{\infty}(\beta)$, we set $s=\beta^{\frac{1}{1-\alpha}} \tilde{s}$ and expand the integrand with respect to $1 /\left(\beta^{\frac{2}{1-\alpha}}\right)$. This yields:

$$
\mathcal{I}^{\infty}(\beta)=\frac{1}{\beta^{\frac{2}{1-\alpha}}} \int_{\beta^{-\frac{1}{1-\alpha}}}^{\infty} \frac{\tilde{s}^{3} \mathrm{~d} \tilde{s}}{\left(\frac{\tilde{s}^{2}}{2}+\tilde{s}^{1+\alpha}\right)^{3}}+O\left(\frac{1}{\beta^{4}}\right) .
$$

We distinguish between three cases:

(I.1) for $\alpha>1 / 3, \quad \mathcal{I}^{\infty}(\beta)=O\left(\frac{1}{\beta^{3}}\right)$;

(I.2) for $\alpha=1 / 3, \quad \mathcal{I}^{\infty}(\beta)=\frac{3}{2} \frac{\ln (\beta)}{\beta^{3}}+O\left(\frac{1}{\beta^{3}}\right)$;

(I.3) for $\alpha<1 / 3, \quad \mathcal{I}^{\infty}(\beta)=\frac{\mu_{\alpha}}{\beta^{\frac{2}{1-\alpha}}}+O\left(\frac{1}{\beta^{3}}\right), \quad \mu_{\alpha}:=\int_{0}^{\infty} \frac{\tilde{s}^{3} \mathrm{~d} \tilde{s}}{\left(\frac{\tilde{s}^{2}}{2}+\tilde{s}^{1+\alpha}\right)^{3}}$.

In $\mathcal{I}^{0}(\beta)$, we set $u=\beta s^{1+\alpha}$ and expand the integrand w.r.t. $1 / \beta^{\frac{1}{1+\alpha}}$. This yields:

$$
\mathcal{I}^{0}(\beta)=\frac{1}{(1+\alpha) \beta^{\frac{4}{1+\alpha}}} \int_{0}^{\beta} \frac{u^{\frac{3-\alpha}{1+\alpha}} \mathrm{d} u}{(1+u)^{3}}+O\left(\frac{1}{\beta^{4}}\right),
$$

with:

(J.1) for $\alpha<1 / 3, \quad \mathcal{I}^{0}(\beta)=O\left(\frac{1}{\beta^{3}}\right)$; 
(J.2) for $\alpha=1 / 3, \quad \mathcal{I}^{0}(\beta)=\frac{3}{4} \frac{\ln (\beta)}{\beta^{3}}+O\left(\frac{1}{\beta^{3}}\right)$;

(J.3) for $\alpha>1 / 3, \quad \mathcal{I}^{0}(\beta)=\frac{\mu_{\alpha}}{\beta^{\frac{4}{1+\alpha}}}+O\left(\frac{1}{\beta^{3}}\right), \quad \mu_{\alpha}:=\frac{1}{1+\alpha} \int_{0}^{\infty} \frac{u^{\frac{3-\alpha}{1+\alpha}} \mathrm{d} u}{(1+u)^{3}}$.

We obtain (3.10) comparing the values of $\mathcal{I}^{0}(\beta)$ and $\mathcal{I}^{\infty}(\beta)$ in the three cases $\alpha<1 / 3, \alpha=1 / 3$ and $\alpha>1 / 3$.

It remains to handle the remainder term $\mathcal{J}(\beta, h)$. As previously, we split it into $\mathcal{J}^{0}(\beta, h)+\mathcal{J}^{\infty}(\beta, h)$ where:

$$
\mathcal{J}^{0}(\beta, h)=\int_{0}^{1} \frac{s^{7} \mathrm{~d} s}{\left(1+\frac{s^{2}}{2}+\beta s^{1+\alpha}\right)^{4}}=O(1), \quad \mathcal{J}^{\infty}(\beta, h)=\int_{1}^{r_{0} / \sqrt{h}} \frac{s^{7} \mathrm{~d} s}{\left(1+\frac{s^{2}}{2}+\beta s^{1+\alpha}\right)^{4}} .
$$

We set $s=\beta^{\frac{1}{1-\alpha}} \tilde{s}$ in the last integral. This yields:

$$
\mathcal{J}^{\infty}(\beta, h) \leq \int_{1 / \beta^{1 /(1-\alpha)}}^{r_{0} /\left(\sqrt{h} \beta^{1 /(1-\alpha)}\right)} \frac{\tilde{s}^{7} \mathrm{~d} s}{\left(\frac{\tilde{s}^{2}}{2}+\tilde{s}^{1+\alpha}\right)^{4}}= \begin{cases}O(|\ln (h)|) & \text { if } \beta \ll 1 \\ O\left(\left|\ln (\beta)+\frac{1-\alpha}{2} \ln h\right|\right) & \text { if } \beta \gg 1 .\end{cases}
$$

\section{REFERENCES}

[1] Y. Achdou, O. Pironneau and F. Valentin, Effective boundary conditions for laminar flows over periodic rough boundaries. J. Comput. Phys. 147 (1998) 187-218.

[2] G. Barnocky and R. H. Davis, The influence of pressure-dependent density and viscosity on the elastohydrodynamic collision and rebound of two spheres. J. Fluid Mech. 209 (1989) 501-519.

[3] A. Basson and D. Gérard-Varet, Wall laws for fluid flows at a boundary with random roughness. Comm. Pure Appl. Math. 61 (2008) 941-987.

[4] L. Bocquet and J. Barrat, Flow boundary conditions from nano-to micro-scales. Soft Matt. 3 (2007) $985-693$.

[5] H. Brenner and R.G. Cox, The resistance to a particle of arbitrary shape in translational motion at small Reynolds numbers. J. Fluid Mech. 17 (1963) 561-595.

[6] D. Bresch, B. Desjardins and D. Gérard-Varet, On compressible Navier-Stokes equations with density dependent viscosities in bounded domains. J. Math. Pures Appl. 87 (2007) 227-235.

[7] M. Cooley and M. O'Neill, On the slow motion generated in a viscous fluid by the approach of a sphere to a plane wall or stationary sphere. Mathematika 16 (1969) 37-49.

[8] R.H. Davis, Y. Zhao, K.P. Galvin and H.J. Wilson, Solid-solid contacts due to surface roughness and their effects on suspension behaviour. Philos. Transat. Ser. A Math. Phys. Eng. Sci. 361 (2003) 871-894.

[9] R.H. Davis, J. Serayssol and E. Hinch, The elastohydrodynamic collision of two spheres. J. Fluid Mech. 163 (2006) 045302.

[10] D. Gérard-Varet, The Navier wall law at a boundary with random roughness. Commun. Math. Phys. 286 (2009) 81-110.

[11] D. Gérard-Varet and M. Hillairet, Regularity issues in the problem of fluid structure interaction. Arch. Rational Mech. Anal. 195 (2010) 375-407.

[12] M. Hillairet, Lack of collision between solid bodies in a 2D incompressible viscous flow. Commun. Partial Differ. Equ. 32 (2007) 1345-1371.

[13] L. Hocking, The effect of slip on the motion of a sphere close to a wall and of two adjacent sheres. J. Eng. Mech. 7 (1973) 207-221.

[14] W. Jäger and A. Mikelić, Couette flows over a rough boundary and drag reduction. Commun. Math. Phys. 232 (2003) $429-455$.

[15] K. Kamrin, M. Bazant and H. Stine, Effective slip boundary conditions for arbitrary periodic surfaces: the surface mobility tensor. Phys. Rev. Lett. 102 (2009).

[16] C. Kunert, J. Harting and O. Vinogradova, Random roughness hydrodynamic boundary conditions. Phys. Rev. Lett. 105 (2010) 016001.

[17] E. Lauga, M. Brenner and H. Stone, Microfluidics: The no-slip boundary condition (2007).

[18] N. Lecoq, R. Anthore, B. Cichocki, P. Szymczak and F. Feuillebois, Drag force on a sphere moving towards a corrugated wall. J. Fluid Mech. 513 (2004) 247-264.

[19] A. Lefebvre, Numerical simulation of gluey particles. ESAIM: M2AN 43 (2009) 53-80.

[20] P. Luchini, Asymptotic analysis of laminar boundary-layer flow over finely grooved surfaces. Eur. J. Mech. B, Fluids 14 (1995) 169-195.

[21] M. O'Neill, A slow motion of viscous liquid caused by a slowly moving solid sphere. Mathematika 11 (1964) 67-74.

[22] M. O'Neill and K. Stewartson, On the slow motion of a sphere parallel to a nearby plane wall. J. Fluid Mech. 27 (1967) $705-724$.

[23] J. Smart and D. Leighton, Measurement of the hydrodynamic surface roughness of noncolloidal spheres. Phys. Fluids 1 (1989) 52-60.

[24] O. Vinogradova and G. Yakubov, Surface roughness and hydrodynamic boundary conditions. Phys. Rev. E 73 (1986) $479-487$. 